\title{
Effect of Control Surface-Fuselage Inertial Coupling on Hypersonic Vehicle Flight Dynamics
}

\author{
Nathan J. Falkiewicz* Scott G. V. Frendreis* and Carlos E. S. Cesnik ${ }^{\dagger}$ \\ Department of Aerospace Engineering, University of Michigan, Ann Arbor, Michigan 48109-2140, USA
}

\begin{abstract}
A simulation framework is developed for assessing the effect of control surface inertial loads on hypersonic vehicle response. The framework is based on a partitioned, timemarching approach in which the fuselage and control surface equations of motion are integrated independently and forces/motion at the interface are exchanged between the two systems at pre-determined time intervals. This approach is advantageous in that it does not require direct coupling of the fuselage and control surface models, and therefore allows for the models to be of dissimilar form. Equations of motion for both the fuselage and control surface are presented and a formulation for coupling the two systems is outlined which includes iterations within each aeroelastic time step. The methodology is applied to a representative hypersonic vehicle elevator control surface model which includes aerothermoelastic effects and is attached to a single degree of freedom oscillator representing the fuselage. Results show that control surface inertial effects lead to a departure of the instantaneous control surface lift force by up to a factor of eight with respect to the static value. Investigation of the system response under a commanded change in control surface deflection angle shows that control surface inertia results in departure of the instantaneous control surface pitching moment by up to a factor of 500 with respect to the static value.
\end{abstract}

\section{Nomenclature}

${ }^{*}$ Ph.D. Candidate, Dept. of Aerospace Engineering, University of Michigan, 1320 Beal Ave, Ann Arbor, MI 48109, Student Member AIAA.

${ }^{\dagger}$ Professor of Aerospace Engineering, Dept. of Aerospace Engineering, University of Michigan, 1320 Beal Ave, Ann Arbor, MI 48109, Associate Fellow AIAA.
$=$ body-fixed reference frame

= generalized damping matrix

$=$ specific heat

$=$ control surface elastic modal coordinates

$=$ modulus of elasticity, Earth-fixed reference frame

$=$ physical load vector

$=$ generalized load vector

$=$ fuselage ordinary natural frequencies

= gravitational acceleration

$=$ Heaviside step function

$=$ coefficient matrices in control surface integration scheme

$=$ altitude

$=$ thickness of $i$-th layer of thermal protection system

$=$ coefficient matrices in fuselage integration scheme

$=$ physical geometric stiffness matrix

$=$ physical conventional stiffness matrix, stiffness of fuselage spring

$=$ physical modified structural stiffness matrix

$=$ generalized modified stiffness matrix

$=$ generalized stiffness matrix

$=$ Lagrangian 
$M \quad=$ physical mass matrix, Mach number, fuselage mass

$M_{y}^{W / B} \quad=$ pitching moment exerted by control surface on fuselage through attachment point

$m \quad=$ generalized mass matrix

$Q_{i} \quad=i$-th generalized force acting on fuselage

$q_{i} \quad=$ arbitrary generalized coordinate

$R \quad=$ residual

$T \quad=$ temperature, total kinetic energy

$\mathcal{T}_{0} \quad=$ uniform initial temperature condition

$t \quad=$ time

tol $=$ convergence tolerance

$U \quad=$ transformation matrix between $x_{u}^{R}$ and $x_{r}$

$V \quad=$ total potential energy

$w_{a} \quad=$ enforced $z$ displacement at control surface attachment

$x \quad=$ physical degrees of freedom

$\alpha \quad=$ vehicle angle of attack

$\alpha_{\text {net }} \quad=$ control surface net angle of attack

$\beta \quad=$ rigid-body translational velocity of fuselage

$\alpha_{T} \quad=$ coefficient of thermal expansion

$\Delta t \quad=$ time step size

$\delta \quad=$ control surface deflection angle

$\zeta \quad=$ rigid-body rotational velocity of fuselage

$\zeta_{\delta} \quad=$ modal damping factor associated with control surface actuator

$\eta \quad=$ structural modal coordinates of fuselage

$\kappa=$ thermal conductivity of material

$\mu_{i} \quad=$ scalar coefficients in time-dependence of enforced motion

$\nu \quad=$ Poisson's ratio

$\rho \quad=$ density of material

$\tau \quad=$ time delay

$\Phi \quad=$ control surface modal matrix

$\omega_{i} \quad=i$-th circular natural frequency

$\omega_{\delta} \quad=$ frequency parameter associated with control surface actuator

Subscripts

$A E \quad=$ aeroelastic

$A T \quad=$ aerothermal

$B \quad=$ body-fixed

$\mathrm{cmd} \quad=$ commanded value

$d \quad=$ time instant at which $\alpha$ is decremented

$f \quad=$ fuselage

HT $\quad=$ heat transfer

$i \quad=$ time instant at which $\alpha$ is incremented

$\max \quad=$ maximum application temperature

$r \quad=$ restrained degrees of freedom

$T=$ component due to thermal loads

$u \quad=$ unrestrained degrees of freedom

$\infty \quad=$ freestream

$\alpha \quad=$ time duration for increment in angle of attack

$\beta \quad=$ generalized load associated with fuselage rigid-body translational velocity

$\zeta=$ generalized load associated with fuselage rigid-body rotational velocity

$\eta \quad=$ generalized load associated with fuselage elastic degrees of freedom

Superscripts

$-1 \quad=$ matrix inverse

A $\quad=$ component of structural loads due to aerodynamic pressure

$C \quad=$ constraint motion

E $\quad=$ elastic displacement 


\section{Introduction}

ESIGN and simulation of hypersonic vehicles (HSVs) require consideration of a variety of disciplines due

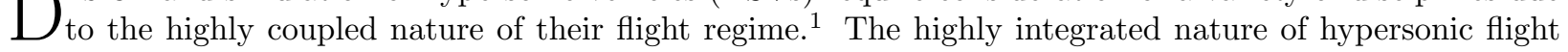
stems from various factors specific to these types of vehicles. Airbreathing hypersonic vehicles typically consist of a tightly integrated airframe along with a scramjet propulsion system. The forward fuselage of the vehicle represents the compression ramp which produces the necessary flow conditions for the inlet of the propulsion system. This results in a pressure distribution which causes a nose-up pitching moment. The aft section of the vehicle consists of an external exhaust nozzle shaped to allow for expansion of the flow exiting the engine. Additionally, the location of the engine below the vehicle center of gravity results a nose-up pitching moment due to thrust which must be balanced. ${ }^{2}$ Further complicating the coupling between the propulsion system and the airframe are the elastic deformations of the forebody and vehicle pitch response which affect the inlet conditions to the engine. ${ }^{3}$ Thus to assess the overall vehicle performance, the effect of flexibility must be considered.

In addition to the effects described above, aerodynamic heating due to flow stagnation and boundary layer friction can also have a significant impact on HSVs. ${ }^{1,4}$ Hypersonic vehicles with airbreathing propulsion systems must fly at relatively low altitudes to maintain the dynamic pressure required for optimal engine performance. ${ }^{4}$ One consequence of this requirement is that the high dynamic pressure and high Reynolds number lead to surface heating becoming a major design driver. The surface heating in turn leads to heat being conducted through the internal vehicle structure. The spatial variation of temperature throughout the structure leads to a change in stiffness distribution through two effects: degradation of material properties due to temperature-dependence and geometric stiffening effects due to internal thermal stresses. The effect of aerodynamic heating on the control surfaces is particularly important to consider as the control surfaces experience a large variation in temperature and the resulting change in stiffness and the deformation due to thermal loads can alter the vehicle flight dynamics.

Flight dynamic simulation of HSVs is further complicated by the large computational expense involved in capturing all of these disciplines and their interactions in a full-order sense. While high-fidelity modeling techniques exist for each of these disciplines, the use of such techniques is computationally infeasible in a vehicle design and simulation setting for such a highly coupled problem. Early in the design stage, many iterations of analyses may need to be carried out as the vehicle design matures, thus requiring quick analysis turn-around time. Additionally, the number of states and number of degrees of freedom used in the analyses must be small enough to allow for efficient control simulation and design. As a result, alternative approaches must be considered for vehicle simulations. There are two methodologies that can be utilized in the generation of low-order models. The first approach is to apply simplifying assumptions that enable the use of firstprinciples models. These models are characterized by their low-order form and they can often be solved analytically, thus preventing the need to time-march the solution. The second approach involves the use of reduced-order models (ROMs) that are derived from high-fidelity analysis tools. Use of high-fidelity tools alone is infeasible due to their high order and long run time. However, by using the output of these tools along with reduced-order modeling techniques, computationally tractable systems of governing equations with low numbers of states can be obtained. In this study, both first-principles models and reduced-order models are used to perform HSV flight dynamics simulations. The aerodynamic heating and unsteady aerodynamic pressures are both computed using first-principles models. The transient thermal and structural dynamic response of the control surface are both computed via reduced-order modeling techniques. Subsequent sections provide the details of the formulations employed in the flight dynamic simulation framework.

The challenges associated with flight dynamics and control analysis of air-breathing HSVs have been reviewed in the literature. ${ }^{5,6}$ To address these challenges, previous research into the flight dynamics of hypersonic vehicles has largely utilized analytical models of the various disciplines. The closed-form nature of these models allows for characterization of the vehicle dynamics early in the design cycle and permits evaluation of stability derivatives more readily than with numerical models. The first effort to develop a comprehensive analytical model was conducted by Chavez and Schmidt. ${ }^{3}$ That work used Newtonian impact 
theory for the aerodynamic pressures, 1D aero/thermo analysis for the propulsion system, and a lumpedmass modal model for the structural dynamics. The methodology was applied to a $2 \mathrm{D}$ hypersonic vehicle geometry in which the control effectors consisted of aerodynamic pitch-control surfaces as well as the engine fuel flow and diffuser area ratio. The derived equations of motion were linearized and analytical expressions were obtained for the stability and control derivatives.

A subsequent work ${ }^{7}$ employed a Lagrangian approach to capture the elastic deformation, fluid flow, rotating machinery, and spherical Earth. The resulting equations of motion governing the rigid body and elastic degrees of freedom were derived and a preliminary study of the significance of selected terms in the equations was presented. A three degree-of-freedom, point-mass dynamic model was also outlined and the equations were presented. For a single-stage-to-orbit configuration, the Coriolis force was found to reach values up to $6 \%$ of the vehicle weight. Another work $^{2}$ presented a nonlinear physics-based model of the longitudinal dynamics for an air-breathing HSV. Oblique shock and Prandtl-Meyer expansion theory were used for the aerodynamics and the structure was modeled as two cantilever beams clamped at the center of mass of the fuselage. The vehicle analyzed in Ref. 2 included an elastic fuselage and elevator control surfaces which were modeled as a rigid flat plates hinged at their mid-chord points. The equations of motion were derived and linearized to assess vehicle stability and coupling between the rigid body and elastic dynamics. Results demonstrated that the linearized aircraft dynamics are unstable and exhibit nonminimum phase behavior in most cases. Coupling between the short-period mode and fuselage bending mode was also exhibited.

In another approach, ${ }^{8}$ a closed form, control-oriented model was obtain by replacing complex force and moment functions from a truth model with curve-fitted approximations. The resulting system was used to demonstrate an example control design based on approximate feedback linearization. The inclusion of additional flexible effects was found to render the original control design ineffective and an additional actuator was needed to enhance the control authority of the vehicle.

While the above papers were successful in developing comprehensive models of hypersonic vehicles for flight dynamic analysis, they did not include the structural dynamics of the control surfaces as well as the resulting inertial coupling between those surfaces and the fuselage. The effect of such coupling can be important as it may result in a complex-conjugate pair of zeros in the elevator-to-pitch rate transfer function, thus altering the speed-of-response of the vehicle. ${ }^{9}$ The goal of the current work is therefore to develop a modeling methodology which includes control surface structural dynamics and is capable of capturing the resulting inertial loads exerted by the control surface on the fuselage of the vehicle.

\section{Simulation Framework}

The simulation framework of the current work is based on a partitioned approach in which the fuselage and control surface equations of motion are integrated separately and information is exchanged between the two at pre-determined time intervals. The advantage of using separate models for the fuselage and control surfaces is that each model can be tailored specific to the physics of interest for that component. In the full vehicle simulation, the equations of motion for the fuselage are derived in analytical form by approximating it as a 1D beam, while those for the control surfaces are based on a finite element discretization. The effect of aerodynamic heating on the control surfaces is expected to be strong in comparison with its effect on the fuselage. As such, aerodynamic heating is only included in the control surface model. Therefore, the fuselage model will be dissimilar in form with respect to the control surface model, and a straighforward monolithic coupling is not easily facilitated. The use of independent models for the fuselage and control surface with information being exchanged between the two at the interface is advantageous in that it does not require a direct coupling between the two components. Additionally, the use of separate models for the fuselage and control surfaces allows for different time-integration schemes and time steps to be employed for each based on the expected time scales of the system dynamics. To couple the structures, interface information must be exchanged between the fuselage and control surfaces at specified intervals. Information is passed to the control surface equations of motion in the form of fuselage accelerations and displacements for the degrees of freedom at the interface. Information is passed back to the fuselage equations of motion in the form of forces exerted by the control surface on the fuselage at the interface.

The aeroelastic framework for the flexible fuselage response has been developed by Frendreis and Cesnik. ${ }^{10}$ A flowchart of this framework is given in Fig. 1. The structural model of the fuselage consists of a structural representation and appropriate boundary conditions. The structural representation is taken to 
be an analytical model based on an Euler-Bernoulli beam. The boundary conditions are chosen to reflect a structure in free flight, such as free-free boundary conditions for a beam. The unsteady aerodynamic model is comprised of a steady shock-expansion component with a piston theory correction to account for unsteady effects. In addition to aerodynamic loads, the fuselage also experiences propulsive loads, which are determined with a scramjet model. Since the control surfaces are not directly included in the fuselage aeroelastic analysis, their contribution is expressed as a set of resultant forces and moments applied at their attachment points. These loads are determined within the control surface aerothermoelastic framework, which is described subsequently. The coupled rigid body/structural equations of motion under loading from unsteady aerodynamics, propulsion, and the control surfaces provide the rigid body and structural responses of the fuselage. These are then used to determine the prescribed root motion of the control surface, which is passed to the control surface aerothermoelastic model.

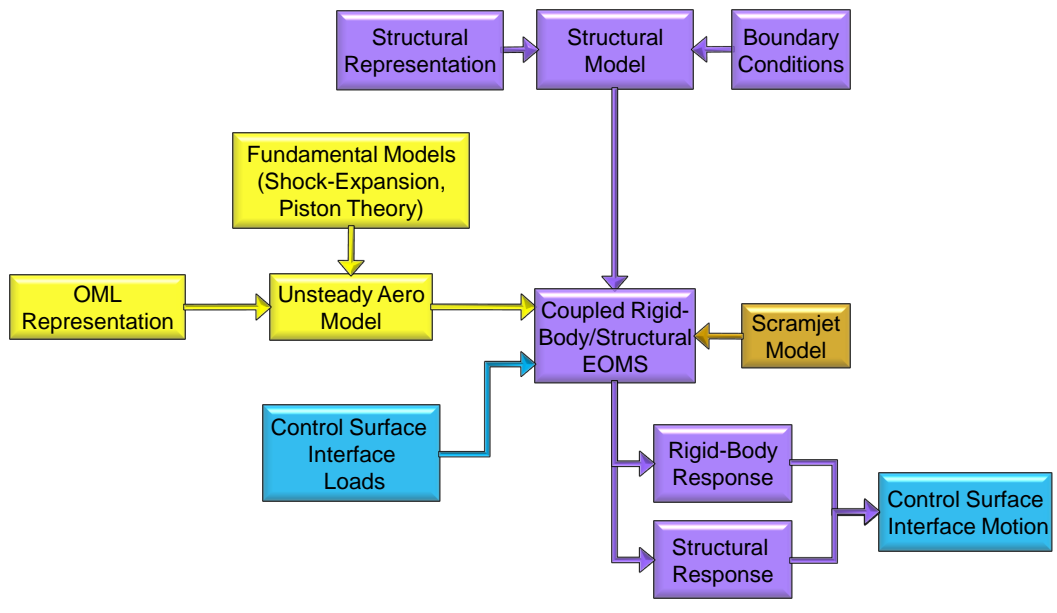

Figure 1. Fuselage aeroelastic simulation framework.

In order to accurately capture the contributions of the control surfaces to the overall vehicle dynamics, aerothermoelastic effects on the control surfaces must be included. The aerothermoelastic model of the control surface used in the present paper is based on a reduced-order modeling framework developed by Falkiewicz and Cesnik. ${ }^{11-15}$ A flowchart of the aerothermoelastic framework used to model the control surfaces is given in Fig. 2. The process begins with the calculation of the heat flux at the outer surface of the structure at initial time using the Eckert reference temperature method. ${ }^{16}$ With the boundary conditions and initial conditions of the thermal problem known, the transient temperature distribution is marched forward in time. Solution of the heat transfer problem is carried out in modal space using modes from POD to avoid the computational cost of running full-order finite element analysis. Bypassing of the full-order thermal solution via the reduced-order solution is indicated by the gray blocks. The structural boundary conditions for the control surface are determined by its layout as well as the fuselage motion at the interface which comes from the solution of the fuselage equations of motion. This framework considers two coupling mechanisms between the thermal solution and the structural stiffness. The first involves the geometric stiffness effects due to thermal stresses that result from thermal expansion of the structure. The second is due to the temperature-dependence of the Young's modulus resulting from the high temperatures experienced in hypersonic flight. In addition to thermal effects on the geometric stiffness, the change in temperature also results in thermal loads being applied to the structure.

With the stiffness and structural loads known, the structural dynamics system of equations in physical space is transformed to a suitable reduced modal basis. The reduced modal system is then solved for the modal coordinates to obtain the structural dynamic response. Once the response is known, it is used to compute the interface loads that the control surface exerts on the fuselage. These interface loads are passed to the fuselage equations of motion and represent force components at the degrees of freedom located at the control surface attachment point. The structural deformations of the control surface couple with the aerothermal problem due to the effect on aerodynamic flow properties, which change the heat flux. The deformations also result in a change in aerodynamic pressures which modify the structural loads. With the deformed configuration known at the current time step, the unsteady aerodynamic flow properties are updated using third-order piston theory and the process is repeated at the next time step. After a pre- 
determined number of aeroelastic iterations have been carried out, the heat flux boundary conditions are recalculated and the thermal solution is updated.

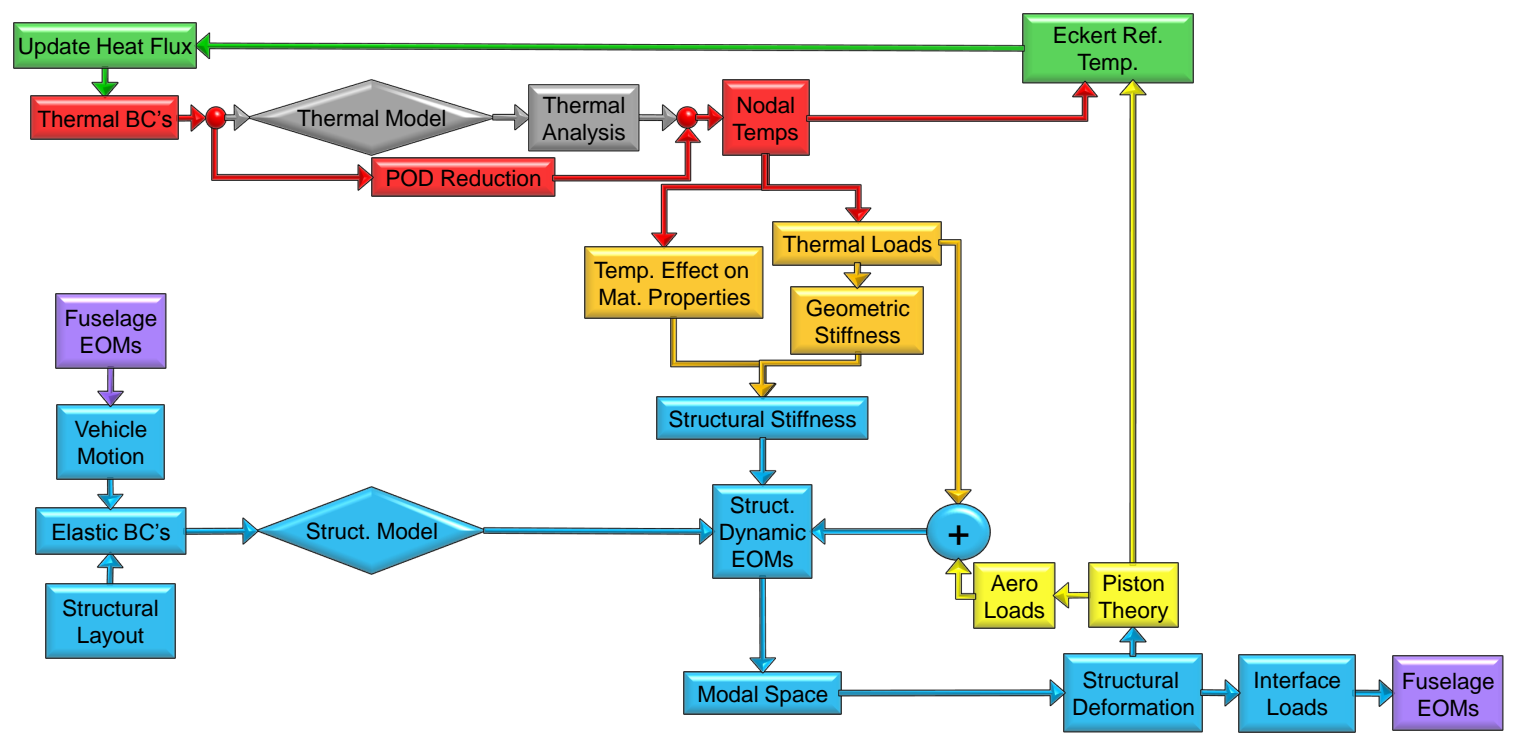

Figure 2. Reduced-order aerothermoelastic modeling framework for control surface model.

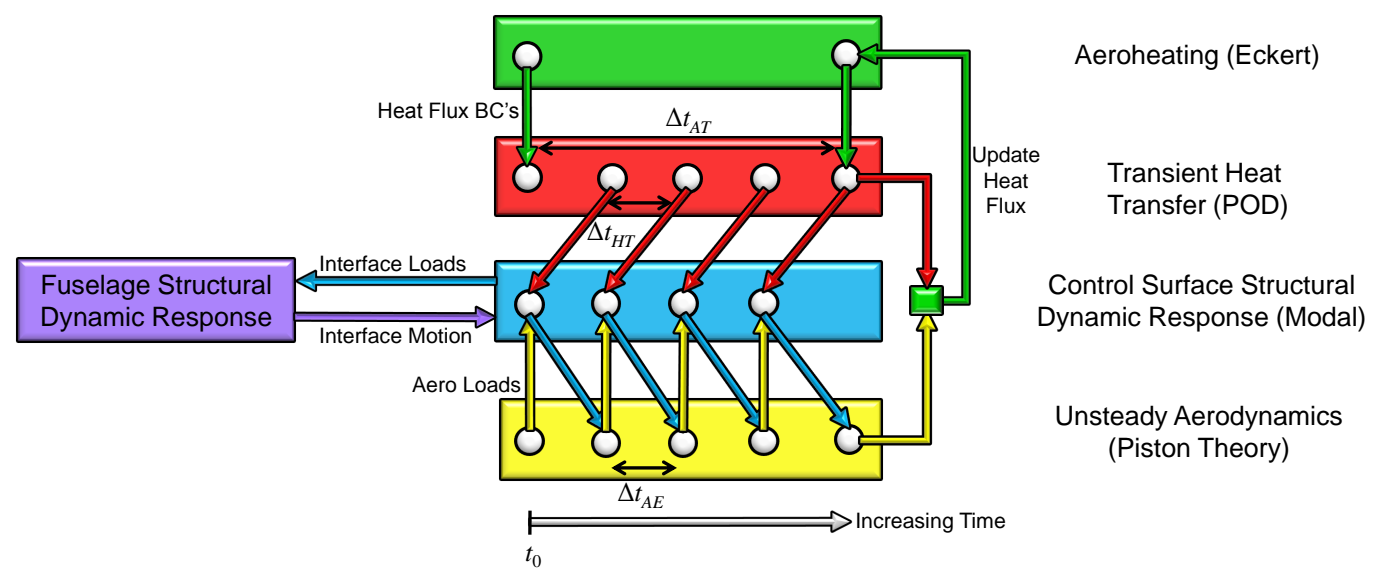

Figure 3. Overview of aerothermoelastic time-stepping schedule for control surface model.

A time-marching procedure with updates to the thermal and structural boundary conditions at specified intervals is utilized for computing the aerothermoelastic response of the control surfaces. An outline of the time-stepping schedule is given in Fig. 3. The size of the aeroelastic time step, $\Delta t_{A E}$, is smaller than the size of the aerothermal time step, $\Delta t_{A T}$, due to the fact that the aeroelastic time scale is faster than the thermal time scale. The procedure begins by calculating the aerodynamic flow properties over the undeformed structure at initial time, $t_{0}$. Using the flow properties, the heat flux at the outer surface is found along with the local skin friction coefficients using the Eckert referece temperature method. With the thermal boundary conditions known, a pre-determined number of thermal time steps are taken, each of size $\Delta t_{H T}$, until the time $t_{0}+\Delta t_{A T}$ is reached. The thermal loads based on the temperature change between $t_{0}$ and $t_{0}+\Delta t_{A E}$ are then applied to the structural configuration at $t_{0}$. Additionally, the aerodynamic loads based on the already calculated flow properties are applied to the structure. The structural dynamic response solution is then marched forward one time step of size $\Delta t_{A E}$. In order to march the control surface structural dynamic solution forward in time, the enforced displacements and accelerations at the interface due to fuselage motion must be specified. However, the fuselage motion in turn depends on the loads that the control surface exerts on the fuselage at the interface. Therefore, within each aeroelastic time step, iterations are performed between the control surface and fuselage in which the control surface model passes interface 
loads to the fuselage, and the fuselage passes back interface motion. The mathematical details of how the iterations are performed are described in a subsequent section. Once the two components have been brought into equilibiurm at time $t_{0}+\Delta t_{A E}$, the converged vector of control surface displacements is stored. These displacements are then fed back into the aerodynamic solver and the flow properties are calculated at time $t_{0}+\Delta t_{A E}$ over the updated deformed configuration. The aeroelastic iterations continue to be carried for a pre-determined number of time steps. Once the time instant $t_{0}+\Delta t_{A T}$ has been reached, the instantaneous flow properties and wall temperatures are used to update the heat flux boundary conditions to the thermal problem. With the updated thermal boundary conditions known, the transient thermal solution is marched forward from the time instant $t_{0}+\Delta t_{A T}$ to the time instant $t_{0}+2 \Delta t_{A T}$ and the process is repeated.

\section{Hypersonic Vehicle Representation}

\section{III.A. Fuselage Model}

There are two components to the representation of the flexible hypersonic vehicle fuselage: the internal structural representation and the exterior OML. The internal structural representation models the elasticity of the aircraft and is used in determining the governing equations of motion for the vehicle. The OML models the vehicle exterior and is used in the unsteady aerodynamic and propulsive analyses. The structural representation for the vehicle can come in a variety of forms (e.g., analytical beam, 3D finite element model, etc.). An analytical beam representation has been implemented ${ }^{10}$ to model the bending and torsion of the hypersonic vehicle fuselage.

The OML is a representation of the exterior of the vehicle that is used in determining the aerodynamic loads. Since the internal structure is able to deform, the OML must be able to deform as well; therefore, an OML consisting of a series of panels, as shown in Fig. 4, is chosen. Each panel in the model is triangular, so that it will remain planar under arbitrary deformations. This specific OML geometry was provided by VSI ${ }^{\mathrm{a}}$ and is described in Ref. 17. The OML includes both the fuselage as well as the scramjet cowl. The recessed region on the top of the fuselage is where a spacecraft would be mounted when the hypersonic vehicle is being used for launch purposes. The lifting surfaces at the aft section of the vehicle are all-movable control surfaces that rotate about a hinge line located at their mid-chord. These surfaces are not considered part of the fuselage structure and are included in Fig. 4 only to illustrate their locations with respect to the fuselage.

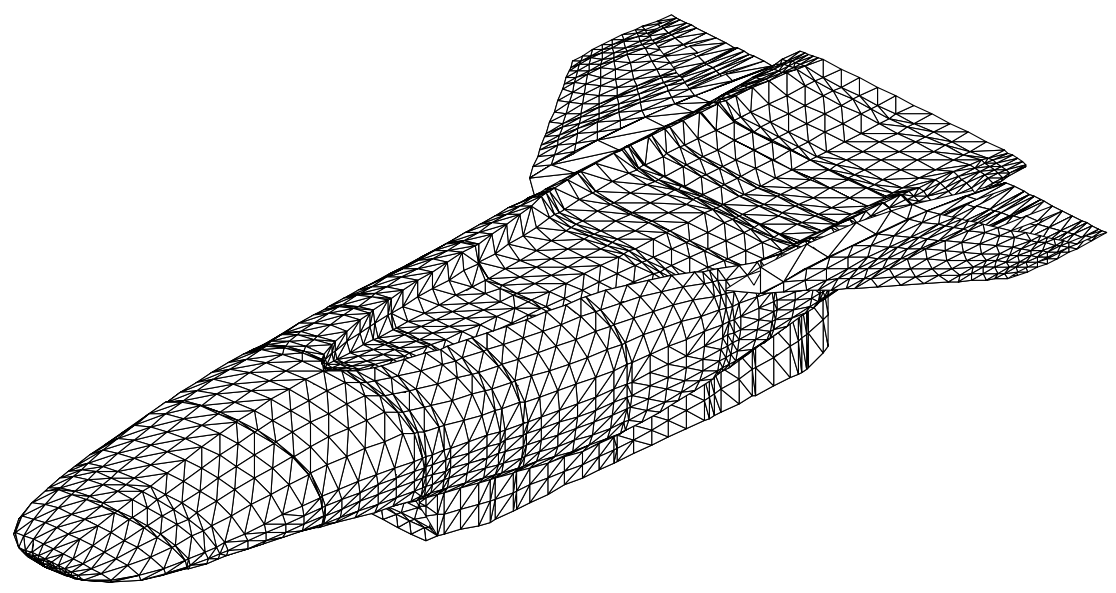

Figure 4. Isometric view of the fuselage outer mold line geometry.

\section{III.B. Control Surface Model}

A finite element model representing the all-movable control surfaces depicted in Fig. 4 has been created for use in this study. The thickness from the top skin layer to the bottom skin layer is $4 \%$ chord length. ${ }^{18}$ The top and bottom skin layers are each equipped with two $3.8 \mathrm{~mm}$ thick thermal protection system layers, and

\footnotetext{
${ }^{\mathrm{a}}$ Vibroacoustics Solutions, Inc. 2214 229th Place Ames, Iowa 50014
} 
thus the thickness of the outer mold line is $4 \%$ chord length plus the $15.2 \mathrm{~mm}$ of thermal protection system material. The chord length at the root is $5.2 \mathrm{~m}$. $(17 \mathrm{ft} .)^{19}$ and the leading edge makes an angle of $34^{\circ}$ with the $y$ axis while the trailing edge makes an angle of $18^{\circ}$ with the $y$ axis. ${ }^{20}$ Planform and cross-sectional views of the airfoil are given in Fig. 5 and Fig. 6, respectively.

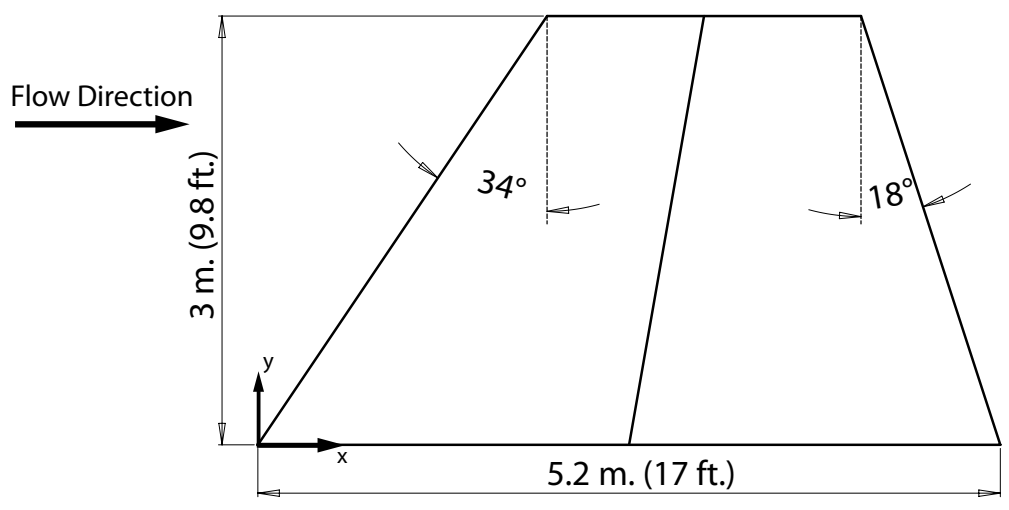

Figure 5. Planform geometry of control surface model.

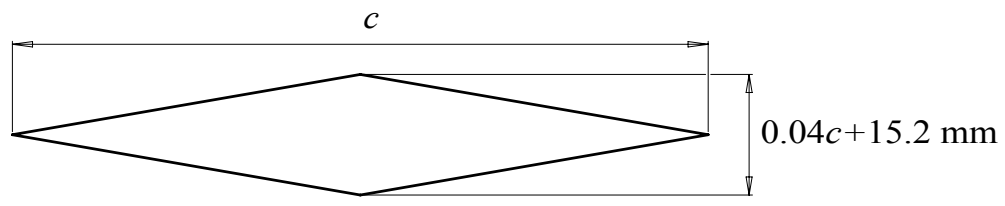

Figure 6. Cross-sectional geometry of control surface model.

A survey of the literature reveals a wide range of design strategies for mitigating the high temperatures experienced in hypersonic flight. ${ }^{21-24}$ This study considers a thermal protection system consisting of an outer heat shield and middle insulation layer on top of the skin as shown in Fig. 7. The material for the heat shield is chosen to be René 41 as it was found to be efficient in terms of mechanical properties at elevated temperatures. For the insulation layer, three different materials were considered in the preliminary materials evaluation: Internal Multiscreen Insulation (IMI), High Temperature Flexible Min-K, and Q-Fiber Felt. Of these, the Min-K insulation, which is a proprietary silica based material faced with Astroquartz cloth ${ }^{25}$ is selected due to its relatively low thermal diffusivity. For the structure (both skin and stiffeners), the Titanium alloy TIMETAL 834 (formerly known as IMI 834) is chosen. The advantage of using this alloy is that its maximum application temperature is $600^{\circ} \mathrm{C}$ compared with that of Ti- $6242 \mathrm{~S}\left(520^{\circ} \mathrm{C}\right)$, Ti- 6242 $\left(450^{\circ} \mathrm{C}\right)$, Ti- $811\left(400^{\circ} \mathrm{C}\right)$, and Ti-6-4 $\left(300^{\circ} \mathrm{C}\right) \cdot{ }^{26}$

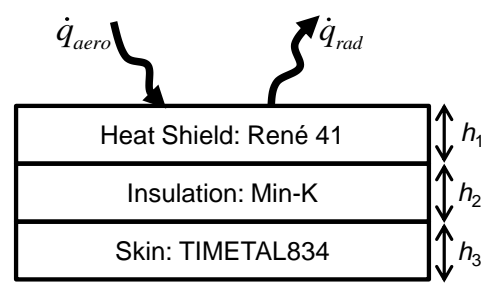

Figure 7. Schematic of material stacking scheme at outer mold line of structure.

The thermal and mechanical properties of the three materials employed in the model are shown in Table 1, where "T-dep." indicates that the property is temperature-dependent. ${ }^{20,25,27,28}$ Note that the temperaturedependent material properties for René 41 are extrapolated beyond the available data and the maximum application temperature, $T_{\max }$, is set to $1500 \mathrm{~K}$. The emissivity of the heat shield is taken to be $0.85 .^{20}$ The thermal strain is calculated based on the temperature change with respect to a reference stress-free 
temperature. The reference temperature used for calculation of thermal strain is taken to be $293 \mathrm{~K}$ for all materials.

Table 1. Structural and thermal material properties used in the study.

\begin{tabular}{l||c|c|c|c|c|c|c|c}
\hline \hline & $\begin{array}{c}\rho \\
{\left[\mathrm{kg} / \mathrm{m}^{3}\right]}\end{array}$ & $\begin{array}{c}E \\
{[P a]}\end{array}$ & $\nu$ & $\begin{array}{c}\alpha_{T} \\
{[\mu \mathrm{m} / \mathrm{m} / \mathrm{K}]}\end{array}$ & $\begin{array}{c}\kappa \\
{[W / m / K]}\end{array}$ & $\begin{array}{c}c_{p} \\
{[\mathrm{~J} / \mathrm{kg} / \mathrm{K}]}\end{array}$ & $\begin{array}{c}T_{\max } \\
K\end{array}$ & $\begin{array}{c}h \\
{[\mathrm{~mm}]}\end{array}$ \\
\hline Heat Shield & 8240 & T-dep. & 0.31 & T-dep. & 18 & 541 & 1500 & 3.8 \\
\hline Insulation & 256 & Neglect & Neglect & Neglect & 0.052 & 858 & 1250 & 3.8 \\
\hline Skin $^{\mathrm{a}}$ & 4550 & T-dep. & 0.31 & 11 & 7 & 525 & 873 & 6.35 \\
\hline \hline
\end{tabular}

a The properties $\nu, \kappa$, and $c_{p}$ for TIMETAL 834 were obtained from http://www.matweb.com/search/DataSheet. aspx?MatGUID=a74096c99aa6486382a9c9e1be0883c4.

The finite element model used for the thermal and structural modeling aspects of the study is shown in Fig. 8 with the top surface removed for visualization purposes. The model consists of the thermal protection system described above along with chordwise and spanwise stiffeners. The material used for the stiffeners is TIMETAL 834 and the thickness of all stiffeners is $25.4 \mathrm{~mm}$ ( $1 \mathrm{in}$ ). The model contains 2,812 thermal degrees of freedom and 8,074 structural degrees of freedom. The heat shield and insulation layer are each modeled using 6-node solid wedge elements while the top and bottom skins and stiffeners are modeled using 3-node, 2-dimensional triangular elements. Of the 6,886 elements in the model, 3,456 are solid elements and 3,430 are triangular elements. The control surface is taken to be all-movable about a hinge line located at the mid-chord ${ }^{19}$ and will thus be connected to the vehicle main body through a torque tube. This attachment is modeled by imposing the condition that control surface translates and rotates with the fuselage at the attachment region indicated by the gray circle in Fig. 8. In addition, the nodes at the root are constrained against translation in the $y$ direction. Because the stiffness of the insulation layer is neglected, rigid (Nastran RBE2) elements are used between each skin node and the corresponding node at the outer surface of the insulation layer to prevent singularities in the solution.

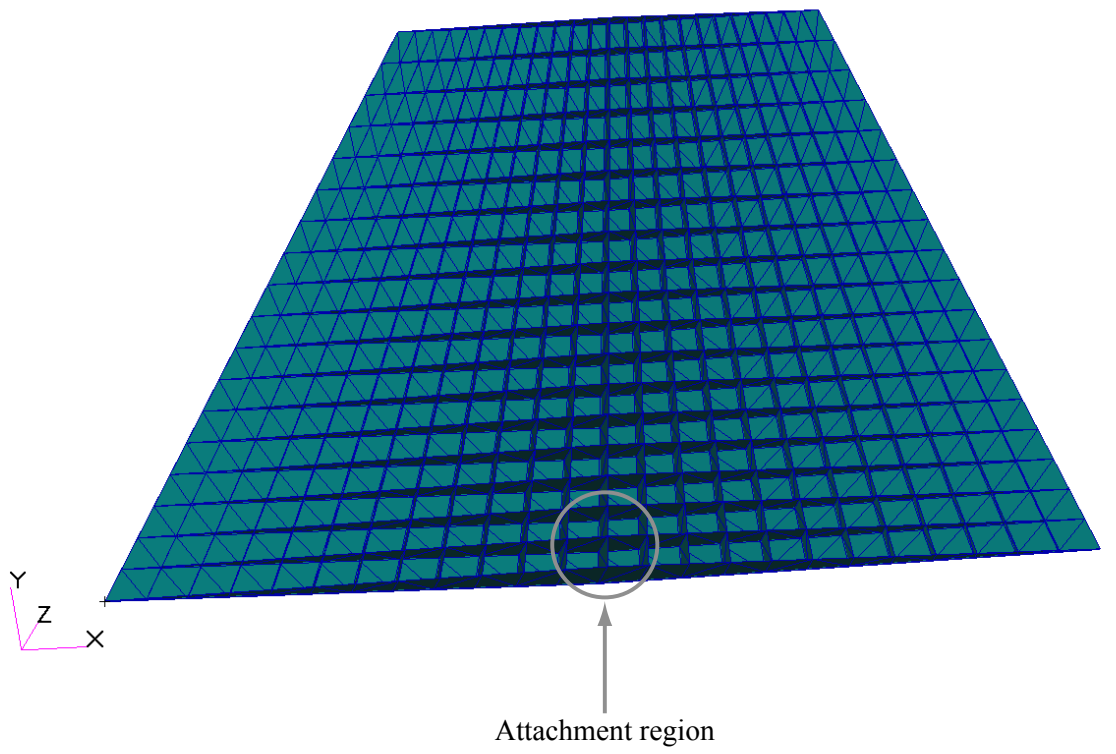

Figure 8. Finite element model of control surface used in study. 


\section{Formulation of Equations of Motion}

\section{IV.A. Fuselage Equations of Motion}

The equations of motion for the flexible hypersonic vehicle fuselage in 3D flight are derived using a Lagrangian approach. This approach is chosen instead of a Newtonian approach because it avoids the need to calculate internal forces within the structure. First, there are two frames of reference that must be introduced: an inertial Earth-fixed reference frame ( $E$ frame) and a moving, body-fixed reference frame ( $B$ frame). Next, the flight variables must be defined. The rigid body translational velocity of the vehicle (i.e., the velocity of the $B$ frame origin with respect to the $E$ frame origin) is represented by $\beta$, and the rigid body rotational velocity is represented by $\zeta$. The structural deformations are expressed in terms of the structural modal coordinates, $\eta$. When the equations of motion are derived, it is assumed that the structural mode shapes are computed a priori. To derive the equations of motion using Lagrange's approach, the total kinetic and potential energies ( $T$ and $V$, respectively) are expressed in terms of the generalized coordinates and velocities, $\eta, \beta$, and $\zeta$, as well as their time derivatives. Then, defining the Lagrangian as $\mathcal{L} \equiv T-V$, the equation of motion governing coordinate $q_{i}$ is given by

$$
\frac{d}{d t}\left(\frac{\partial \mathcal{L}}{\partial \dot{q}_{i}}\right)-\frac{\partial \mathcal{L}}{\partial q_{i}}=Q_{i},
$$

where $Q_{i}$ is the generalized force corresponding to $q_{i}$. The equations of motion can then be assembled into a matrix equation of the form

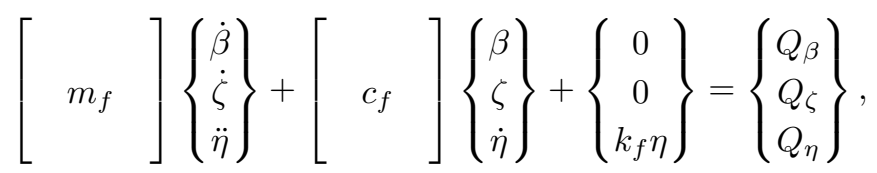

where $m_{f}$ is the generalized mass matrix, $c_{f}$ is the generalized damping matrix, and $k_{f}$ is the generalized stiffness matrix. The mass and damping are not constant; e.g., the mass matrix will change throughout flight as fuel is burned. The damping matrix, which contains the gyroscopic acceleration terms, is dependent on the rigid body rotational velocity, $\zeta$, and will also vary as the vehicle inertia changes. The details of this derivation of the equations of motion as applied to the free-free beam structure are available in Ref. 10.

Once the equations of motion are assembled, the next task is to determine the generalized forces, $Q_{\beta}$, $Q_{\zeta}$, and $Q_{\eta}$. For the hypersonic vehicle fuselage, there are four sources of external loading: aerodynamics, propulsion, gravity, and resultant forces from the lifting surfaces that include the control surfaces in this particular partition. Since the vehicle is undergoing unsteady rigid body motion, as well as structural deflections, an unsteady aerodynamic model is required. The aerodynamic model used for the fuselage is based on a steady shock-expansion analysis with an unsteady correction computed using piston theory. These models are applied in a local inclination scheme, i.e., each panel of the OML, as shown in Fig. 4, is considered separately, and the aerodynamic load on that panel is a function of its total velocity and orientation only.

The thrust required for hypersonic flight is provided by a scramjet engine. Many previous efforts in hypersonic vehicle simulation have relied on a simplified scramjet propulsion model, ${ }^{29}$ which uses quasi-1D flow relations. An improved scramjet model ${ }^{30}$ has been recently made available. This model analyzes both the internal flowpath of the engine and the external inlet and nozzle. The external inlet and nozzle are analyzed with 2D inviscid flow relations and include the effects of chemistry. The internal flowpath/combustor uses a quasi-1D flow model that includes effects such as mixing. Since this overall model is essentially 2D, it is applied to a 3D vehicle in a strip-theory scheme, as shown in Fig. 9, where the dark blue lines represent the $2 \mathrm{D}$ engine profiles analyzed by the propulsion code. The $2 \mathrm{D}$ profiles are determined by tracing from points at the edge of the engine cowl forward or backward along the OML. While only three profiles are shown in Fig. 9, more may be used.

The effect of the control surfaces on the fuselage dynamics is expressed as a set of resultant forces and moments at the control surface attachment points. The resultant forces and moments are determined by passing the fuselage motion at the control surface attachment point to the control surface equations of motion. Using this specified root motion, the resultant forces and moments are calculated by the control surface aerothermoelastic model. These resultant forces include contributions from both the aerodynamic loading and the unsteady structural response of the control surfaces. As described in section III.B, the control surfaces are treated as all-movable surfaces about a hinge line located at the mid-chord. Deflection of the control surfaces by the control system is therefore represented as enforced rotation about this line. 


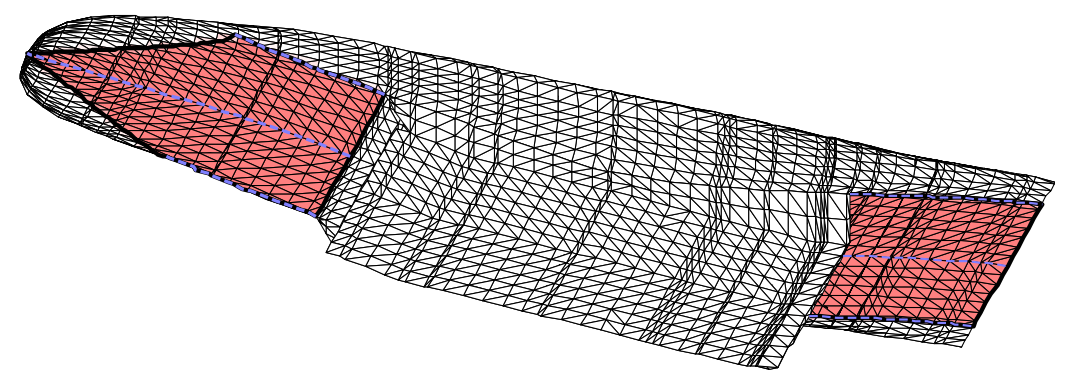

Figure 9. Outer mold line panel kinematics.

\section{IV.B. Control Surface Equations of Motion}

The usage of the Eckert reference temperature formulation used to calculate the aerodynamic heating over the control surface, the POD formulation for obtaining the transient temperature distribution of the control surface, and the third-order piston theory formulation for the control surface aerodynamic flow calculation are presented in a previous work ${ }^{31}$ and are thus omitted here for brevity. Therefore, the emphasis of the current section is on the solution for the structural dynamic response of the control surface subjected to thermal and unsteady aerodynamic loads as well as enforced displacements and accelerations due to fuselage motion.

For a control surface with prescribed accelerations and displacements at degrees-of-freedom (DOFs) $r$, the equations of motion can be partitioned as

$$
\left[\begin{array}{ll}
M_{r r} & M_{r u} \\
M_{u r} & M_{u u}
\end{array}\right]\left\{\begin{array}{l}
\ddot{x}_{r} \\
\ddot{x}_{u}
\end{array}\right\}+\left[\begin{array}{ll}
K_{r r}^{*}(T) & K_{r u}^{*}(T) \\
K_{u r}^{*}(T) & K_{u u}^{*}(T)
\end{array}\right]\left\{\begin{array}{l}
x_{r} \\
x_{u}
\end{array}\right\}=\left\{\begin{array}{c}
F_{r} \\
F_{u}^{H}+F_{u}^{A}
\end{array}\right\},
$$

where $M$ is the physical mass matrix, $x(t)$ are the physical degrees of freedom, $F^{H}(T)$ is the load vector due to heating, $F^{A}(t)$ is the load vector due to aerodynamic pressure, the subscript $r$ corresponds to the restrained DOFs (those with prescribed accelerations and displacements), and the subscript $u$ corresponds to the unrestrained DOFs (those without prescribed accelerations and displacements). The modified stiffness matrix, $K^{*}(T)$, is given by

$$
K^{*}(T) \equiv K(T)+K_{G}(T),
$$

where $K(T)$ is the conventional stiffness matrix that varies due to the temperature-dependence of the material properties and $K_{G}(T)$ is the geometric stiffness matrix resulting from thermal stresses. In this formulation, the equations of motion for the unrestrained control surface DOFs are cast in terms of the elastic displacements relative to the constraint motion caused by the enforced displacements at the restrained DOFs. The term "constraint motion" refers to the displacements that the structure would undergo if the prescribed motion was applied statically and inertial effects were not present. Note that the term "constraint motion" is specifically used instead of "rigid body motion" because the number of DOFs with prescribed motion is greater than that required to constrain rigid body motion in this case. Such a formulation is advantageous because the constraint motion is accounted for separately and the equations of motion are associated only with the elastic response, thus the structural modal matrix does not need to be modified to include constraint modes. Therefore the control surface structural modal matrix is composed only of elastic modes in this formulation. The first step is to calculate the constraint motion due to enforced motion at the unrestrained DOFs, denoted by $x_{u}^{C}$. This quantity is obtained by neglecting inertial loads and external loads in the second row of Eq. (3) and solving for $x_{u}$, i.e.,

$$
x_{u}^{C}=-\left(K_{u u}^{*}\right)^{-1} K_{u r}^{*} x_{r} .
$$

Note that a transformation, $U$, can be defined in Eq. (5) between $x_{u}^{C}$ and $x_{r}$ such that

$$
x_{u}^{C}=U x_{r}, \text { where } U=-\left(K_{u u}^{*}\right)^{-1} K_{u r}^{*} .
$$


If the number of DOFs with prescribed motion were exactly equal to the minimum number of DOFs required to constrain rigid body motion, the columns of $U$ would represent rigid body modes. Because in this case the number of DOFs with prescribed motion is greater than that required to constrain rigid body motion, the columns of $U$ represent constraint modes.

The next step is to derive the equations governing the elastic deformation of the unrestrained DOFs, $x_{u}^{E}$, relative to the constraint motion. Expanding Eq. (3), one obtains

$$
\begin{aligned}
M_{r r} \ddot{x}_{r}+M_{r u} \ddot{x}_{u}+K_{r r}^{*} x_{r}+K_{r u}^{*} x_{u} & =F_{r} \\
M_{u r} \ddot{x}_{r}+M_{u u} \ddot{x}_{u}+K_{u r}^{*} x_{r}+K_{u u}^{*} x_{u} & =F_{u}^{H}+F_{u}^{A} .
\end{aligned}
$$

Recall that the total motion of the unrestrained DOFs is the sum of the constraint motion plus the elastic motion, i.e.,

$$
x_{u}=x_{u}^{C}+x_{u}^{E} .
$$

Substituting Eq. (8) into Eq. (7b), one obtains

$$
M_{u r} \ddot{x}_{r}+M_{u u}\left(\ddot{x}_{u}^{C}+\ddot{x}_{u}^{E}\right)+K_{u r}^{*} x_{r}+K_{u u}^{*}\left(x_{u}^{C}+x_{u}^{E}\right)=F_{u}^{H}+F_{u}^{A},
$$

and using Eq. (5) in Eq. (9), the system becomes

$$
M_{u r} \ddot{x}_{r}+M_{u u}\left[-\left(K_{u u}^{*}\right)^{-1} K_{u r}^{*} \ddot{x}_{r}+\ddot{x}_{u}^{E}\right]+K_{u r}^{*} x_{r}+K_{u u}^{*}\left[-\left(K_{u u}^{*}\right)^{-1} K_{u r}^{*} x_{r}+x_{u}^{E}\right]=F_{u}^{H}+F_{u}^{A} .
$$

Bringing all terms associated with the restrained DOFs to the right-hand side of Eq. (10), the equation becomes

$$
M_{u u} \ddot{x}_{u}^{E}+K_{u u}^{*} x_{u}^{E}=-M_{u r} \ddot{x}_{r}+M_{u u}\left(K_{u u}^{*}\right)^{-1} K_{u r}^{*} \ddot{x}_{r}-K_{u r}^{*} x_{r}+K_{u u}^{*}\left(K_{u u}^{*}\right)^{-1} K_{u r}^{*} x_{r}+F_{u}^{H}+F_{u}^{A},
$$

and simplifying the right-hand side of Eq. (11) results in

$$
M_{u u} \ddot{x}_{u}^{E}+K_{u u}^{*} x_{u}^{E}=\left[M_{u u}\left(K_{u u}^{*}\right)^{-1} K_{u r}^{*}-M_{u r}\right] \ddot{x}_{r}+F_{u}^{H}+F_{u}^{A} .
$$

The relation given by Eq. (12) is the system to be solved for the relative elastic motion of the unrestrained DOFs, $x_{u}^{E}$. Note that the solution to Eq. (12) requires only the accelerations of the restrained DOFs, $\ddot{x}_{r}$, and not the displacements. However, $x_{r}$ is required to compute the constraint motion, $x_{u}^{C}$. Due to the large number of degrees of freedom, direct solution of Eq. (12) within the aerothermoelastic control surface framework is not desirable. A common approach to reduce the order of such a system is to employ a modal transformation in which the structural displacements are expressed as a linear combination of a small number of basis vectors which are the free vibration mode shapes of the structure. However, this approach cannot be applied directly for Eq. (12) as the mode shapes change over time due to modification of the stiffness from geometric stiffness and material degradation effects. The approach taken in this work is to first perform an off-line calculation and select a reduced number of Ritz modes based on free vibration modes and loaddependent Ritz vectors ${ }^{32}$ evaluated at a reference thermal state. These Ritz modes are then used as the modal basis for solution of the structural response throughout the simulation. This procedure is applicable as the Ritz modes need only to satisfy the geometric boundary conditions, ${ }^{33}$ which will always be the case regardless of the stiffness distribution. The modal matrix containing the structural reference modes, $\Phi$, will not be updated throughout the simulation, thus preventing the need to solve an eigenvalue problem of the full system during the course of the simulation. Though the reference modes will not be updated throughout the simulation, the stiffness matrix will be updated each time the structural dynamic response is calculated to account for temperature-dependent material properties and geometric stiffening. Updating of the conventional stiffness matrix is performed using the temperature-dependence of the material properties of the various materials. The geometric stiffness matrix is updated by solving a static finite element problem based on the thermal loads from temperatures at the current time step and the material coefficients of thermal expansion. As discussed previously, an important result of solving only for the elastic response in Eq. (12) is that the structural basis must only contain elastic modes. Because the remainder of the motion is accounted for in Eq. (8), the structural modal matrix need not contain constraint modes.

The reduced-order system is obtained by first representing the elastic motion, $x_{u}^{E}(t)$, as a linear combination of the Ritz modes such that

$$
x_{u}^{E}(t)=\Phi d(t),
$$


where $d$ represents the modal coordinates of the Ritz modes which are stored as columns of the modal matrix, $\Phi$. Note that since the number of Ritz modes used in the modal expansion is much less than the number of physical degrees of freedom in the model, the computational cost of the solution is reduced. Once the modified stiffness matrix is known at the current time instant, the system is reduced by substituting Eq. (13) into Eq. (12) and pre-multiplying the system by $\Phi^{T}$ to project the system onto the basis, i.e.,

$$
\Phi^{T} M_{u u} \Phi \ddot{d}(t)+\Phi^{T} K_{u u}^{*}(T) \Phi d(t)=\Phi^{T}\left(\left[M_{u u}\left(K_{u u}^{*}(T)\right)^{-1} K_{u r}^{*}(T)-M_{u r}\right] \ddot{x}_{r}(t)+F_{u}(t, T)\right),
$$

where the net force, $F_{u}(t, T)$, is defined as

$$
F_{u}(t, T) \equiv F_{u}^{H}(T)+F_{u}^{A}(t)
$$

The generalized mass matrix, $m_{u u}$, generalized stiffness matrix, $k_{u u}^{*}$, and generalized net force vector, $f_{u}$, are then identified from Eq. (14) as

$$
\begin{aligned}
m_{u u} & =\Phi^{T} M_{u u} \Phi \\
k_{u u}^{*}(T) & =\Phi^{T} K_{u u}^{*}(T) \Phi \\
f_{u}(t, T) & =\Phi^{T} F_{u}(t, T),
\end{aligned}
$$

and the reduced system in modal form is given as

$$
m_{u u} \ddot{d}(t)+k_{u u}^{*}(T) d(t)=f_{u}(t, T)+\Phi^{T}\left(\left[M_{u u}\left(K_{u u}^{*}(T)\right)^{-1} K_{u r}^{*}(T)-M_{u r}\right] \ddot{x}_{r}(t)\right)
$$

As the mass of the structure is taken to be constant in this work, the reference modes are orthogonal with respect to the mass matrix and the generalized mass matrix, $m_{u u}$, reduces to the identity matrix. Since the modified stiffness matrix is continuously changing due to transient heating, we have no guarantee of orthogonality of the reference modes with respect to stiffness, and the equations are coupled. As such, the reduced-order system of equations in modal space is integrated numerically to calculate $d(t)$ at each aeroelastic time step. The numerical integration method employed is similar to the Newmark- $\beta$ method except that the load vector is averaged over three time instants and the stiffness matrix is modified such that the dynamic equation of motion reduces to a static solution if no inertial effects or damping exist. ${ }^{34}$ The scheme uses a central finite difference representation for the velocity and acceleration at discrete times, given by ${ }^{34}$

$$
\begin{aligned}
& \dot{d}^{(n)}=\frac{d^{(n+1)}-d^{(n-1)}}{2 \Delta t_{A E}} \\
& \ddot{d}^{(n)}=\frac{d^{(n+1)}-2 d^{(n)}+d^{(n-1)}}{\Delta t_{A E}^{2}},
\end{aligned}
$$

where the superscript $(n)$ refers to the time level. The initial conditions, $d^{(0)}$ and $\dot{d}^{(0)}$, are used to generate the vectors $d^{(n-1)}, f_{u}^{(n-1)}$, and $f_{u}^{(n)}$ for the initial time step, $n=0$, using

$$
\begin{aligned}
d^{(-1)} & =d^{(0)}-\dot{d}^{(0)} \Delta t_{A E} \\
f_{u}^{(-1)} & =k_{u u}^{*} d^{(-1)} \\
f_{u}^{(0)} & =k_{u u}^{*} d^{(0)} .
\end{aligned}
$$

Note that this formulation assumes that the initial acceleration for all points is zero (initial velocity is constant). In order to maintain consistency with the central difference approximation for the modal accelerations, the enforced acceleration in Eq. $(17), \ddot{x}_{r}(t)$, is approximated at time level $(n)$ using a central difference formula, i.e.,

$$
\ddot{x}_{r}(t)=\frac{x_{r}^{(n+1)}-2 x_{r}^{(n)}+x_{r}^{(n-1)}}{\Delta t_{A E}^{2}} .
$$

Substituting the finite difference approximations of the velocities and accelerations, Eqs. (18) and (20), into the equations of motion, Eq. (17), and averaging the applied loads over three adjacent time instants, the equations of motion are re-written as

$$
H_{1} d^{(n+1)}=H_{2}+H_{3} d^{(n)}+H_{4} d^{(n-1)},
$$


where,

$$
\begin{aligned}
H_{1} & =\frac{1}{\Delta t_{A E}^{2}} m_{u u}+\frac{1}{3} k_{u u}^{*} \\
H_{2} & =\frac{1}{3}\left(f_{u}^{(n+1)}+f_{u}^{(n)}+f_{u}^{(n-1)}\right)+\Phi^{T}\left[M_{u u}\left(K_{u u}^{*}(T)\right)^{-1} K_{u r}^{*}(T)-M_{u r}\right] \frac{x_{r}^{(n+1)}-2 x_{r}^{(n)}+x_{r}^{(n-1)}}{\Delta t_{A E}^{2}} \\
H_{3} & =\frac{2}{\Delta t_{A E}^{2}} m_{u u}-\frac{1}{3} k_{u u}^{*} \\
H_{4} & =\frac{-1}{\Delta t_{A E}^{2}} m_{u u}-\frac{1}{3} k_{u u}^{*} .
\end{aligned}
$$

The vector of structural modal coordinates at the end of the time step, $d^{(n+1)}$, is obtained by decomposing $H_{1}$ and applying it to the right-hand side of Eq. (21). Once $d^{(n+1)}$ is obtained, the total motion of the unconstrained degrees of freedom in physical space is computed via Eqs. (5), (8), and (13) using

$$
x_{u}^{(n+1)}=-\left(K_{u u}^{*}\right)^{-1} K_{u r}^{*} x_{r}^{(n+1)}+\Phi d^{(n+1)} .
$$

Once the displacements of the unrestrained DOFs are known at a given time $t$, the force contribution due to control surface (wing) motion, $F_{r}^{W}$, can be calculated at time $t$ by computing the quantity $M_{r u} \ddot{x}_{u}+K_{r u}^{*} x_{u}$ from the first row of Eq. (3) and moving it to the right-hand side to treat as a forcing function acting on the fuselage at the interface. This force contribution is computed using,

$$
F_{r}^{W}(t, T)=-M_{r u} \frac{x_{u}^{(n+1)}-2 x_{u}^{(n)}+x_{u}^{(n-1)}}{\Delta t_{A E}^{2}}-K_{r u}^{*} \frac{x_{u}^{(n+1)}+x_{u}^{(n)}+x_{u}^{(n-1)}}{3},
$$

where the accelerations of the unrestrained DOFs, $\ddot{x}_{u}$, are calculated using a central difference and the displacements are averaged over three adjacent time levels in order to maintain consistency with the numerical integration scheme. Once $F_{r}^{W}(t, T)$ is known, it can then be passed to the fuselage equations of motion in order to update the loads. In order to compute the net external force that the control surface (wing) exerts relative to the fuselage (body), $F_{r}^{W / B}$, the elastic motion of the unrestrained control surface DOFs relative to the interface DOFs is utilized. Therefore, $F_{r}^{W / B}$ is given by

$$
F_{r}^{W / B}=-K_{r u}^{*}\left(x_{u}^{E}-x_{u, T}^{E}\right),
$$

where $x_{u, T}^{E}$ is the elastic deformation caused by thermal loads. Note that $x_{u, T}^{E}$ must be substracted from $x_{u}^{E}$ because thermal loads are internal to the system and do not result in external forces being exerted on the fuselage.

\section{IV.C. Methodology for Fuselage-Control Surface Coupling}

As described previously, the flight dynamics simulation framework for the HSV is based on a partitioned approach in which the fuselage and control surface equations of motion are solved independently and information is exchanged between the two at pre-determined intervals. The equations of motion for the fuselage and the control surface are presented above, and thus the final step of the formulation is to couple the two systems. Time-marching for the complete vehicle is complicated due to the mutual interdependence of the fuselage and control surface equations of motion. As the focus of this work is on the effect of loads exerted by the control surface at the attachment point, the fuselage representation described in Section III.A is replaced by a point mass attached to a spring which is then connected to the control surface for the purpose of the current study. This configuration is depicted in Fig. 10. Note that the motion of the fuselage mass is constrained such that it it is only permitted to translate in the $z$ direction. Therefore, the enforced motion at the control surface attachment location only contains a $z$ translational component. The attachment between the fuselage mass and the control surface is modeled by a rigid element (Nastran RBE2) that transfers the fuselage motion to the control surface DOFs located approximately within the attachment region shown in Fig. 8. Physically, the spring stiffness, $K_{f}$, represents the bending stiffness of the fuselage as well as the associated aerodynamic stiffness. The goal is to march the complete system in time by modeling the control surface and fuselage (point mass) independently and exchanging information between the two within each time step. 


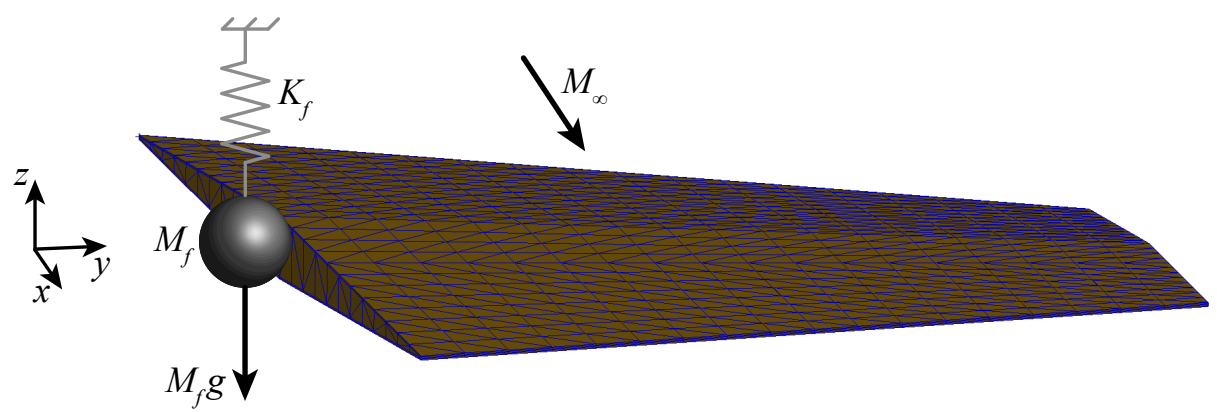

Figure 10. Representation of control surface and fuselage system.

Referring to Eq. (3), the fuselage motion at the interface in this representation is given by

$$
M_{r r} \ddot{x}_{r}(t)+K_{r r} x_{r}(t)=F_{r}+F_{r}^{W}(t, T)
$$

where $M_{r r}$ and $K_{r r}$ are the mass and stiffness associated with the restrained DOFs, $x_{r}(t)$ is the degree of freedom corresponding to the fuselage displacement, and $F_{r}^{W}(t, T)$ is the force exerted by the control surface on the fuselage given in Eq. (24). The force component $F_{r}$ represents the force due to the weight of the fuselage and is given by,

$$
F_{r}=M_{f} g
$$

where $g$ is the gravitational acceleration. For uniformity, the same numerical integration scheme used for the control surface is also used for the fuselage, such that the fuselage displacement at the end of each aeroelastic time step, $(n+1)$, can be found from

$$
I_{1} x_{r}^{(n+1)}=I_{2}+I_{3} x_{r}^{(n)}+I_{4} x_{r}^{(n-1)},
$$

where,

$$
\begin{aligned}
I_{1} & =\frac{1}{\Delta t_{A E}^{2}} M_{r r}+\frac{1}{3} K_{r r} \\
I_{2} & =\frac{1}{3}\left(F_{r}^{(n+1)}+F_{r}^{(n)}+F_{r}^{(n-1)}\right)+F_{r}^{W} \\
I_{3} & =\frac{2}{\Delta t_{A E}^{2}} M_{r r}-\frac{1}{3} K_{r r} \\
I_{4} & =\frac{-1}{\Delta t_{A E}^{2}} M_{r r}-\frac{1}{3} K_{r r} .
\end{aligned}
$$

Note that $F_{r}^{W}$ is already averaged over three adjacent time steps in Eq. (24), and therefore only $F_{r}$ must be averaged in Eq. (29b).

The effect of the control surface response on the fuselage response can be seen by inspecting Eq. (28). Specifically, note that $I_{2}$ is a function of $F_{r}^{W}$, and $F_{r}^{W}$ depends on $x_{u}^{(n+1)}$. Also, recall that $x_{u}^{(n+1)}$ is expressed in terms of the control surface modal coordinates, $d^{(n+1)}$. As shown in Eq. $(22 \mathrm{~b}), d^{(n+1)}$ in turn depends on $x_{r}^{(n+1)}$. We can therefore state that $d^{(n+1)}$ is a function of $x_{r}^{(n+1)}$. To highlight these dependencies, we rewrite Eq. (28) as

$$
I_{1} x_{r}^{(n+1)}=I_{2}\left(d^{(n+1)}\left(x_{r}^{(n+1)}\right)\right)+I_{3} x_{r}^{(n)}+I_{4} x_{r}^{(n-1)} .
$$

Solution of Eq. (30) is complicated due to the fact that $d^{(n+1)}$ is unknown, and depends on the solution to the fuselage response. In a manner analogous to Eq. (30), Eq. (21) for the control surface can be rewritten as

$$
H_{1} d^{(n+1)}=H_{2}\left(x_{r}^{(n+1)}\left(d^{(n+1)}\right)\right)+H_{3} d^{(n)}+H_{4} d^{(n-1)},
$$

where the dependence of $H_{2}$ on both the control surface and fuselage states at time $(n+1)$ is explicitly shown. 
While both the fuselage and control surface systems could be combined into one (as shown in Eq. (3)) and solved monolithically, the goal of this work is to develop a methodology for time-marching multiple coupled systems of differential equations by treating each independently and exchanging appropriate information in a mathematically robust manner. The motivation for such an approach is due to the fact that the HSV will consist of multiple components, each of which will have its own model. As the models are likely to be of dissimilar form, direct monolithic coupling of the models is not easily facilitated. The philosophy of this work is therefore to treat each model as a black-box with respect to the other. Mathematically, this means that $I_{2}\left(d^{(n+1)}\left(x_{r}^{(n+1)}\right)\right)$ in Eq. (30) and $H_{2}\left(x_{r}^{(n+1)}\left(d^{(n+1)}\right)\right)$ in Eq. (31) are treated as unknown functions of $x_{r}^{(n+1)}$ and $d^{(n+1)}$ where information about these functions can only be obtained by evaluating them at specific input values.

Due to the unknown nature of $H_{2}$ and $I_{2}$, Eqs. (30) and (31) are each treated as if they are nonlinear in the other system's states (i.e., the fuselage system is treated as if it is nonlinear in the control surface states, and the control surface system is treated as if it is nonlinear in the fuselage states). Such an assumption preserves the generality of the method such that it is applicable to any two systems of differential equations that are nonlinearly coupled. Furthermore, the methodology is formulated such if the two systems are only linearly coupled, the linear solution is recovered in only one iteration. Due to the assumption of nonlinear coupling, the equations are solved using an iterative approach to bring the two systems to equilibrium at the end of each time step. As opposed to a pseudo-time-marching strategy in which the two components are successively marched in pseudo-time until the equilibrium state is reached, this work formulates the algorithm as a solution to a nonlinear root-finding problem and uses derivative information of the nonlinear function in order to converge to the equilibrium state more efficiently. A secant method is used in this work to increment the state at each iteration due to its property of being superlinearly locally convergent. ${ }^{35}$

Before implementing the methodology, it must be decided which system will be the driving system and which system will be the driven system. The driving system is the one in which the secant method is used to compute the increment to the state at each iteration until convergence is achieved. The state vector for the driven system at time $(n+1)$ is merely updated by marching forward one step based on the state vector from the driving system at time $(n+1)$ for each iteration. Assuming that the driving system is chosen to be the fuselage system, we begin by moving all terms in Eq. (30) to the left-hand side and equating it to the residual at iteration $k, R\left(d_{k}^{(n+1)}\left(x_{r, k}^{(n+1)}\right)\right)$, i.e.,

$$
I_{1} x_{r, k}^{(n+1)}-I_{2}\left(d_{k}^{(n+1)}\left(x_{r, k}^{(n+1)}\right)\right)-I_{3} x_{r}^{(n)}-I_{4} x_{r}^{(n-1)}=R\left(d_{k}^{(n+1)}\left(x_{r, k}^{(n+1)}\right)\right) .
$$

Note that according to Eq. (30), the right-hand side of Eq. (32) should be equal to zero. However, this condition is only achieved when the control surface and fuselage are brought into equilibrium at the end of the time step, $(n+1)$. At intermediate iterations prior to reaching the equilibrium state, the left-hand side of Eq. (32) will be equal to a nonzero residual that is a function of the fuselage and control surface states. The process of bringing the control surface and fuselage to equilibrium is mathematically equivalent to driving the residual to zero in Eq. (32) by iterating on the fuselage state vector, $x_{r}^{(n+1)}$.

A summary of the algorithm used to bring the fuselage and control surface systems to equilibrium within each time step is given in Table 2. The iteration procedure begins with two initial guesses to the fuselage state at the end of the time step, $x_{r, 0}^{(n+1)}$ and $x_{r, 1}^{(n+1)}$, where the subscripts 0 and 1 refer to the iteration number. For each initial guess, $H_{2}$ is computed at step 3 using Eq. (22b) and Eq. (21) is solved for the control surface state vector, $d_{k}^{(n+1)}$, at step 4 . The total control surface displacements in physical space, $x_{u}^{(n+1)}$, are then computed using Eq. (23) in step 5. Once the motion of the control surface at time level $(n+1)$ is known, the loads imparted on the fuselage by the control surface at time level $(n+1)$ are calculated using Eq. (24) to give $F_{r}^{W}(t, T)$ in step 6. With these loads known, $I_{2}$ is computed from Eq. (29b). Using $I_{2}$, the left-hand side of Eq. (32) is evaluated giving the residual at step 8. At this point, if the residual is above the specified tolerance, an iteration loop begins in which the fuselage state is incremented using the fuselage states and residual values at the two previous iterations via the secant method. Iterations are carried out repeating steps $3-8$ as described above, until the residual drops below the specified tolerance. At that point, the fuselage and control surface have been brought into equilibrium at the end of the current time step. The states for each component are then stored, the simulation marches to the next time step, and 
the iteration process is again carried out until equilibrium is achieved at the next time step. This process continues until the simulation is complete.

Table 2. Iteration scheme used for bringing control surface and fuselage into equilibrium at each time step.

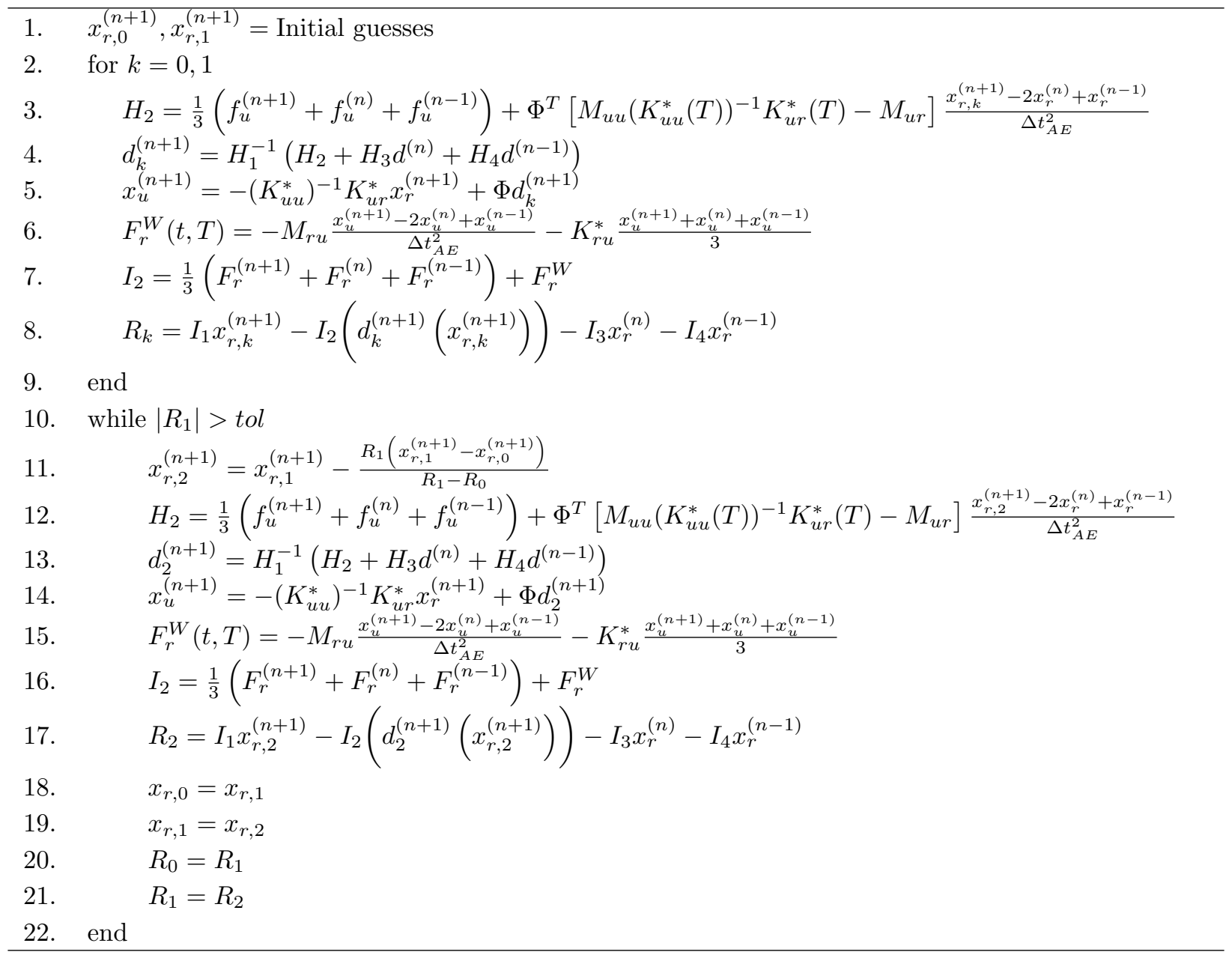

A schematic of the information flow for the iteration scheme is given in Fig. 11, where the numbers in boxes correspond to the step numbers in Table 2. The process begins at point A by calculating the increment to the fuselage state and updating it at the next iteration and moving to point B. Based on the new fuselage displacement, the accelerations of the DOFs at the control surface attachment point are found. These accelerations are passed to control surface equations of motion at point C. Using these boundary conditions, the control surface state vector at the next iteration is computed, therefore moving to point $\mathrm{D}$. Once the solution of the control surface equations of motion is known at point $\mathrm{D}$, the loads exerted by the control surface on the fuselage are updated and passed back to the fuselage system at point A. The fuselage state is again incremented, except this time using the new loads from the control surface. At this point the process repeats and iterations are carried out until the forces exerted by the control surface on the fuselage are brought into equilibrium with the accelerations of the fuselage at the interface location.

\section{Aerothermoelastic Control Surface ROM Generation}

Prior to carrying out simulations, the thermal and structural dynamic ROMs to be used in the control surface framework must be created. As some of the ROMs used in the control surface aerothermoelastic simulation process are dependent on components of other ROMs, these models must be generated in a specific order. An overview of the process is given in Fig. 12. The green blocks in the figure describe the process for generating a kriging-based aerothermal ROM developed by Crowell et al. ${ }^{36}$ and incorporated 


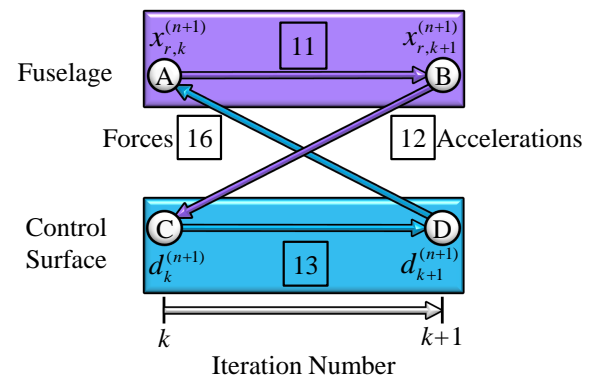

Figure 11. Schematic of fuselage-control surface coupling scheme.

into the aerothermoelastic ROM framework in a recent work. ${ }^{14}$ Because the current work is focused on the structural dynamic and thermal ROM components of the control surface, the Eckert reference temperature method is utilized in place of the aerothermal ROM here. However, the aerothermal ROM component is included in Fig. 12 to illustrate the complete ROM generation process. The first ROM to be created is the POD model for the transient thermal component of the solution. To begin, the range of vehicle flight parameters is defined. Based on these parameters, representative simulations are defined for use in extracting the POD snapshots. Because the aerothermal ROM is dependent on the thermal ROM, the Eckert reference temperature method is used to calculate the heat flux in these simulations. Additionally, the structural dynamic ROM is dependent on the thermal ROM, and thus the high-fidelity structural model is used in these simulations. The accuracy of the thermal ROM is evaluated by running representative aerothermoelastic simulations using both the thermal ROM and full-order model and comparing their output. If greater accuracy is desired, more snapshots are taken and the thermal ROM is updated.

Once an accurate thermal ROM has been created, the next step is to identify the reference thermal state at which to evaluate the structural reference modes. The philosophy used in this work is to take the reference thermal state to be the average nodal temperatures over the thermal snapshots. Based on the frequency range of interest, a set of free vibration modes are evaluated at the reference thermal state including both material property degradation with temperature and geometric stiffening due to thermal stresses. While it is important to include free vibration modes in the basis, they have no association with the spatial distribution of the applied structural loads. Additionally, in cases that are dominated by thermal loads, the structural response is close to quasi-static and the extent to which the free vibration modes are excited is minimal. Therefore, the set of free vibration is augmented by inserting into the modal matrix additional vectors calculated using the method of load-dependent Ritz vectors. ${ }^{32}$ The goal of including load-dependent Ritz vectors is to enrich the basis such that it can capture motion that does not lie in the space of the free vibration modes. A detailed discussion of the load-dependent Ritz vector methodology is given in a previous work. ${ }^{15}$

Once the modal matrix has been assembled, kriging ROMs of the generalized stiffness matrix and physical thermal load vector are created for efficient updating of these quantities as a function of temperature. Specifically, these ROMs are used to directly update $k_{u u}^{*}(T)=\Phi^{T} K_{u u}^{*}(T) \Phi$ and $F_{u}^{H}(T)$ shown in Eqs. (14) and (15), respectively. However, due to the large number of DOFs in the structural model, $K_{u u}^{*}(T)$ and $K_{u r}^{*}(T)$ on the right-hand sides of Eqs. (5) and (14) are approximated by evaluating them at the reference thermal state and holding them constant throughout the simulation. To generate the kriging ROMs, bounds on the POD modal coordinates are established and kriging training cases are run. Note that the formulation and validation associated with the kriging ROMs of the stiffness and thermal loads are given in a previous work. ${ }^{15}$ Once the ROMs are genereated, the accuracy of the structural ROM is assessed by again running representative aerothermoelastic simulations and comparing the output of the structural ROM with that of the full-order structural model. If greater accuracy is desired, the structural modal basis is updated and the process is repeated.

The thermal and structural ROMs are then used in the aerothermal ROM generation process developed by Crowell et al. ${ }^{36}$ An added benefit to expressing the temperature distribution and deformation as a linear combination of modes is that it allows for parameterization of each in terms of a small number of design variables for the purpose of creating the aerothermal ROM. Bounds on the aerothermal ROM parameters are first established. Parameters for the aerothermal ROM consist of the structural and thermal modal coordinates as well as flight parameters such as angle of attack, Mach number, and altitude. Because the 
feasible number of parameters in the aerothermal ROM is limited, a subset of thermal and structural modes are used in its creation. Training cases are set up within the parameter space using Latin Hypercube Sampling (LHS). For each training case, CFD analysis is used to generate training data of the aerodynamic heating. Using this training data, a kriging ROM representing the aerodynamic heat flux at the outer surface is generated. The accuracy is then evaluated using a separate set of evaluation cases. If greater accuracy is desired, more kriging sample points are added and the process is repeated. Once a satisfactory aerothermal ROM has been obtained, the ROM generation process is complete and the thermal, structural, and aerothermal ROMs can then be used for efficient aerothermoelastic simulation.

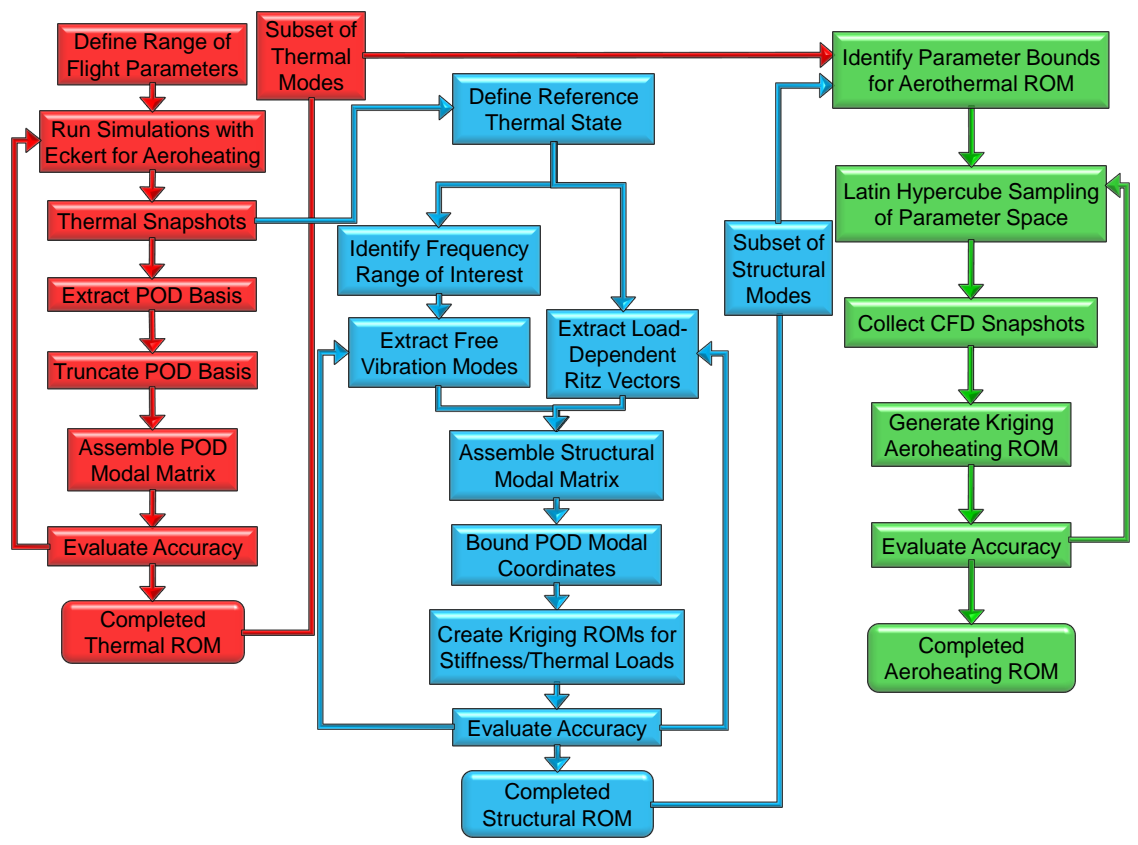

Figure 12. Flowchart of aerothermoelastic ROM generation process.

The aerothermoelastic control surface ROM used in the current work is based on that created in a previous work. ${ }^{15}$ The ranges in flight conditions used in ROM generation for that work are given in Table 3 , where $M_{\infty}$ is the freestream Mach number, $\alpha_{\text {net }}$ is the net control surface angle of attack, $h$ is the altitude, and $\mathcal{T}_{0}$ is the uniform initial temperature condition. The thermal snapshots were based on 10 parallel aerothermoelastic simulations, resulting in a total of 49,510 thermal snapshots. Based on the POD eigenvalues, 32 POD modes were retained for the thermal ROM. Comparison of the thermal ROM to the full-order thermal model for one representative simulation showed that the $L_{\infty}$ error remained below $3 \%$ throughout the complete time range considered.

Table 3. Bounds on flight conditions for aerothermoelastic control surface ROM.

\begin{aligned} & \hline \hline 5.0$\leq M_{\infty} \leq 8.0 \\ & 0.0^{\circ} \leq \alpha_{\text {net }} \leq 4.0^{\circ} \\ & 25.0 \mathrm{~km} \leq h \leq 45.0 \mathrm{~km} \\ & 293 \mathrm{~K} \leq \mathcal{T}_{0} \leq 1500 \mathrm{~K} \\ &$\hline \hline\end{aligned}

The current work makes use of the thermal ROM that was generated in Ref. 15. However, additional studies are conducted in this paper to identify a robust structural basis under thermal, aerodynamic, and base excitation loads. For the purpose of ROM assessment, the time-dependence of the enforced displacements in the $z$ direction at the control surface attachment point, $w_{a}(t)$, is prescribed as

$$
w_{a}(t)=\sum_{i=1}^{3} \mu_{i} \sin \left(\omega_{i} t\right)
$$

where $\mu_{i}$ are real scalar coefficients and $\omega_{i}$ are circular frequencies representing the first three fuselage bending 
natural frequencies. These frequencies are computed based on vehicle properties given in a previous work. ${ }^{10}$ Table 4 gives the circular natural frequencies along with the corresponding values of the ordinary natural frequencies, $f_{i}$, in $\mathrm{Hz}$ as well as the scalar coefficients.

Table 4. Parameters used in time-dependence of enforced motion at control surface attachment point.

\begin{tabular}{ccc}
\hline \hline$i$ & $\omega_{i}[\mathrm{rad} / \mathrm{sec}] / f_{i}[\mathrm{~Hz}]$ & $\mu_{i}$ \\
\hline 1 & $6.9 / 1.1$ & 0.015 \\
2 & $43.2 / 6.9$ & 0.015 \\
3 & $121.1 / 19.3$ & 0.015 \\
\hline \hline
\end{tabular}

In order to determine to appropriate number of free vibration modes and load-dependent Ritz vectors to include in the structural basis, full-order and reduced-order simulations are performed under combined base excitation loads, thermal loads, and unsteady aerodynamic loads at the following flight conditions: $M_{\infty}$ $=6.5, \alpha_{\text {net }}=2^{\circ}, h=35 \mathrm{~km}$. The thermal load vector used in the simulations is held constant over time and is obtained based on the temperatures at the end of a $400 \mathrm{~s}$ aerothermal simulation beginning from room temperature at these flight conditions. By including both base excitation loads and thermal loads in the simulations, the ability of the basis to capture both highly unsteady response due to base motion as well as static response due to thermal deformation can be examined. Based on simulation results, the structural basis is chosen to consist of ten free vibration modes evaluated at the reference thermal state and five additional load-dependent Ritz vectors. The reference thermal state is obtained by averaging the 49,510 thermal snapshots taken in Ref. 15. Of the ten free vibration modes employed in the basis, the natural frequency of the lowest frequency mode is $25 \mathrm{~Hz}$, while that of the highest frequency mode is $186 \mathrm{~Hz}$. Each of the five load-dependent Ritz vectors correspond to a static solution under a representative applied load. The applied load for each Ritz vector is chosen based on a POD analysis of the structural load vector which was carried out in Ref. 15 in order to identify the dominant spatial components of the structural loads.

Recall that two additional approximations are made in the structural dynamic ROM besides the use of the modal subset. One involves the kriging approximations used to directly update the generalized stiffness matrix, $k_{u u}^{*}(T)$, and physical thermal load vector, $F_{u}^{H}(T)$, in Eqs. (14) and (15) as a function of temperature. The other involves the fact that $K_{u u}^{*}(T)$ and $K_{u r}^{*}(T)$ on the right-hand sides of Eqs. (5) and (14) are evaluated at the reference thermal state and held constant throughout the simulation. In order to distinguish the errors due to these approximations from the errors due to modal truncation, three different cases are simulated as shown in Table 5 .

Table 5. Summary of three cases used in structural dynamic ROM evaluation.

\begin{tabular}{cccccc}
\hline \hline Case & Structural Model & $k_{u u}^{*}(T)$ & $F_{u}^{H}(T)$ & $K_{u u}^{*}(T)$ & $K_{u r}^{*}(T)$ \\
\hline E1 & Full-order & N/A & Actual $T /$ Exact & Actual $T /$ Exact & Actual $T /$ Exact \\
E2 & 15-mode ROM & Actual $T /$ Exact & Actual $T /$ Exact & Actual $T /$ Exact & Actual $T /$ Exact \\
E3 & 15-mode ROM & Actual $T /$ Kriging & Actual $T /$ Kriging & Ref. $T /$ Exact & Ref. $T /$ Exact \\
\hline \hline
\end{tabular}

In the table, "Actual $T$ " indicates that a quantity is evaluated at the actual thermal state of the structure, while "Ref. $T$ " indicates that the quantity is evaluated at the reference thermal state obtained by averaging the thermal snapshots. The term "Exact" in the table refers to the fact that no approximation is made when assembling the matrix or vector, while "Kriging" indicates that the corresponding kriging approximation is used. For case E1, the full-order structural model is used and all quantities are evaluated exactly at the actual thermal state. The full-order model does not require the generalized stiffness matrix as indicated by "N/A" in the third column for case E1. For this case, the physical stiffness matrix, $K_{u u}^{*}$, on the left-hand side of Eq. (12) is evaluated exactly at the actual thermal state. Case E2 uses the structural ROM with all quantities evaluated exactly at the actual thermal state. The structural ROM is also used for case E3 and $k_{u u}^{*}(T)$ and $F_{u}^{H}(T)$ are again evaluated at the actual thermal state. However, for this case, these quantities are computed using the corresponding kriging approximations which provide improved computatinal efficiency. Additionally for case E3, $K_{u u}^{*}(T)$ and $K_{u r}^{*}(T)$ on the right-hand sides of Eqs. (5) and (14) are evaluated at the reference thermal state and held constant throughout the simulation. Therefore, the difference between cases E1 and E2 will demonstrate the error incurred solely due to the use of the modal subset in the structural ROM. The difference between cases E2 and E3 will illustrate the error incurred solely due to the kriging 
approximations of the generalized stiffness and physical thermal loads in addition to that due to evaluating $K_{u u}^{*}(T)$ and $K_{u r}^{*}(T)$ on the right-hand sides of Eqs. (5) and (14) at the reference thermal state instead of the actual thermal state. Note that case E3 represents the structural dynamic model that will be used in the remainder in the paper.

Plots of the $z$-direction displacements from cases E1 - E3 for a duration of $1 \mathrm{~s}$ are given at four different nodes in Fig. 13. Though there exist minor discrepancies at certain peaks, the results given in Fig. 13 provide evidence that the structural dynamic control surface ROM represented by case E3 is capable of capturing the full-order response represented by case E1 with reasonable accuracy. Therefore, the 15-mode structural dynamic ROM of the control surface as described above is used in the remainder of this study.

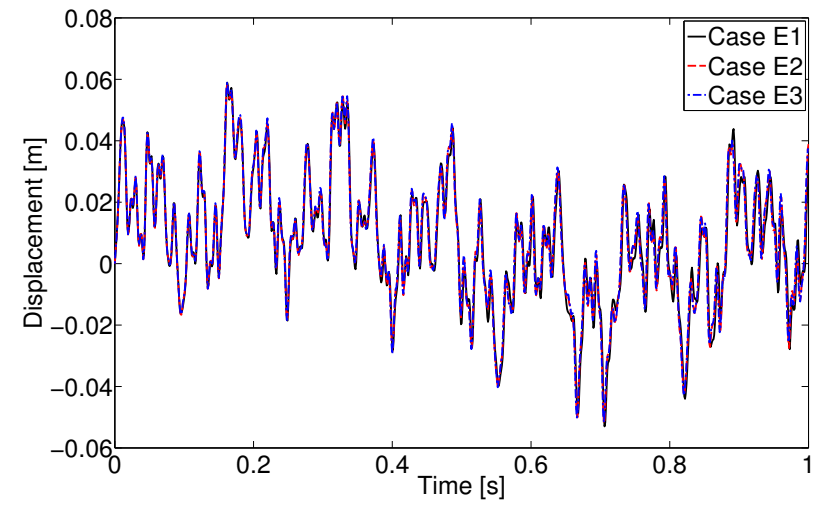

(a) Node 2 (bottom surface, root leading edge).

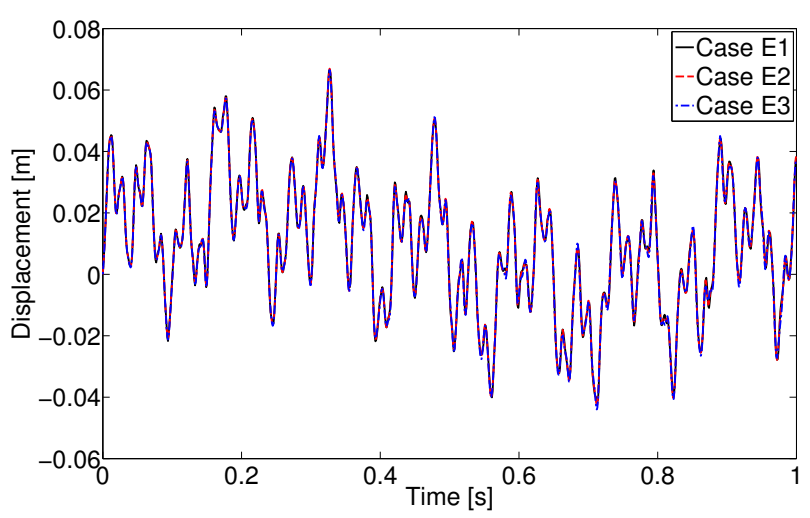

(c) Node 458 (bottom surface, root trailing edge).

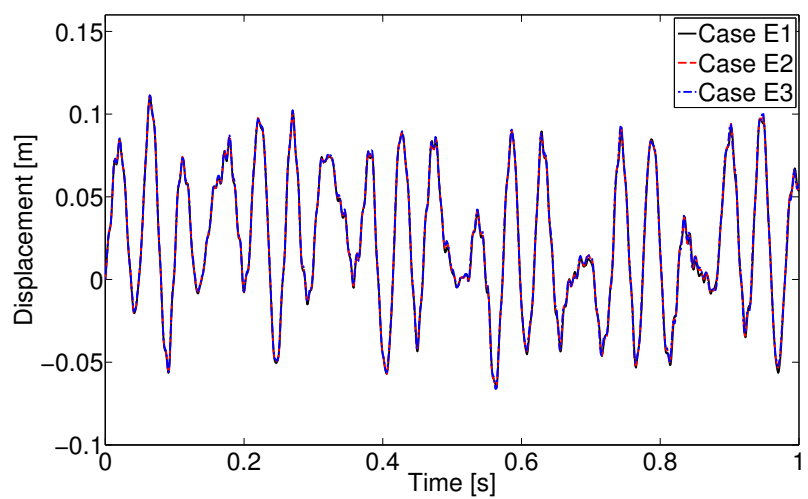

(b) Node 37 (bottom surface, tip leading edge).

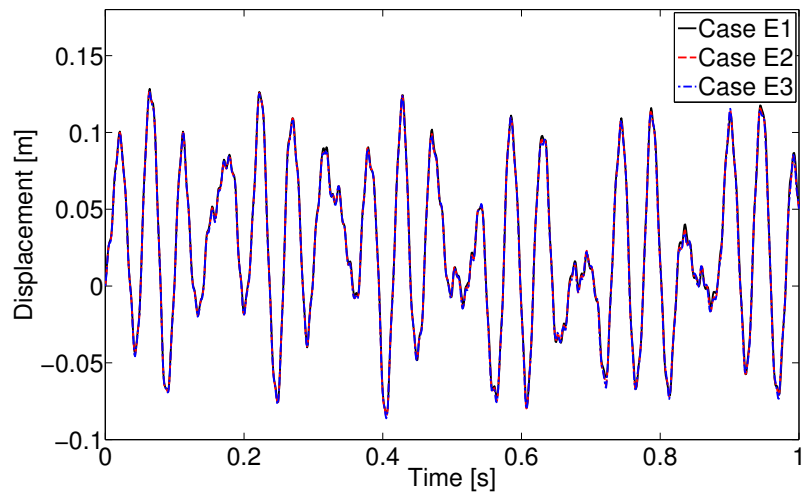

(d) Node 475 (bottom surface, tip trailing edge).

Figure 13. Time-history of $z$ displacements at four selected nodes for cases E1 - E3.

\section{Control Surface-Fuselage Coupling: Results}

\section{VI.A. Validation of Partitioned Solution Methodology}

The fuselage-control surface configuration depicted in Fig. 10 is now used to illustrate the methodology. Before utilizing the partitioned fuselage and control surface models, the partitioned solution methodology is validated against a full-order monolithic solution performed within Nastran. For the validation, the fuselage mass is taken to be $222,222 \mathrm{~kg}$ based on vehicle properties given in a previous work. ${ }^{10}$ The stiffness of the spring associated with the fuselage is chosen such that the natural frequency of the fuselage system is $10 \mathrm{~Hz}$, and thus the spring stiffness is $8.77 \times 10^{8} \mathrm{~N} / \mathrm{m}$. Three validation cases, denoted P1, P2, and P3, are set up to validate the approach, where "P" denotes that these cases are used to validate the partitioned solution methodology. A summary of these cases is given in Table 6. Case P1 uses the monolithic system with the full-order structural model and represents the reference case. Case P2 uses the partitioned approach with the full-order structural model of the control surface, while case P3 uses the partitioned approach with the previously described 15-mode structural ROM of the control surface. The difference between cases P1 and P2 illustrates the error due to use of the partitioned approach, while the difference between cases P2 and P3 
illustrates the error due to the use of the control surface structural ROM. Note that for all three cases, the aerodynamic loads are omitted from the analysis, and the thermal loads are held constant based on those obtained after heating the structure for $400 \mathrm{~s}$ from room temperature. The aeroelastic time step sizes for both the fuselage and the control surface are chosen to be $0.001 \mathrm{~s}$. The simulation is started by releasing the mass representing the fuselage from its equilibrium position at initial time. As the fuselage mass begins to oscillate, it in turn excites the control surface and induces structural dynamic oscillations.

Table 6. Summary of three cases used to validate partitioned solution methodology.

\begin{tabular}{ccc}
\hline \hline Case & Fuselage-Control Surface Coupling & Control Surface Structural Model \\
\hline P1 & Monolithic & Full-order \\
P2 & Partitioned & Full-order \\
P3 & Partitioned & 15-mode ROM \\
\hline \hline
\end{tabular}

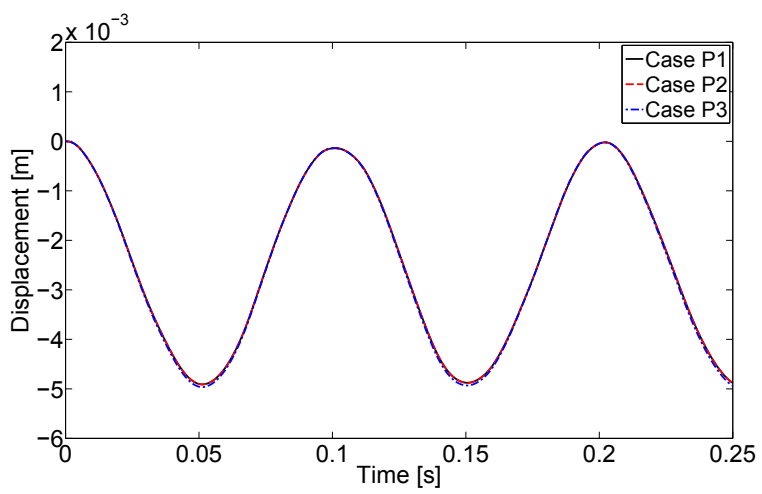

(a) Fuselage node.

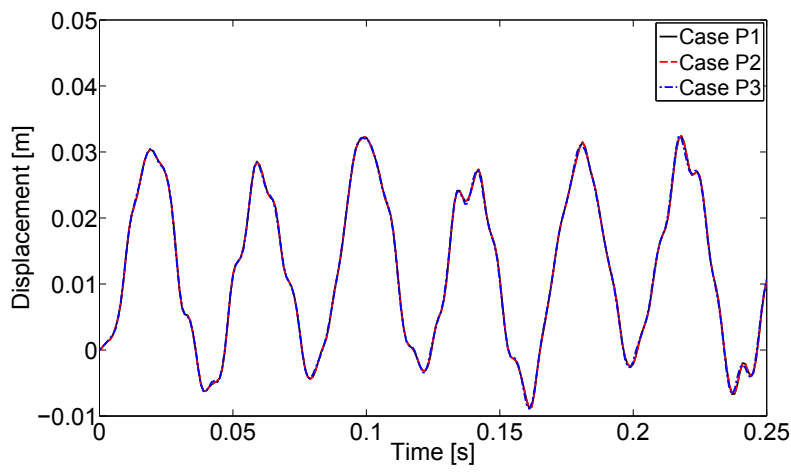

(c) Node 247 (bottom surface, tip mid-chord).

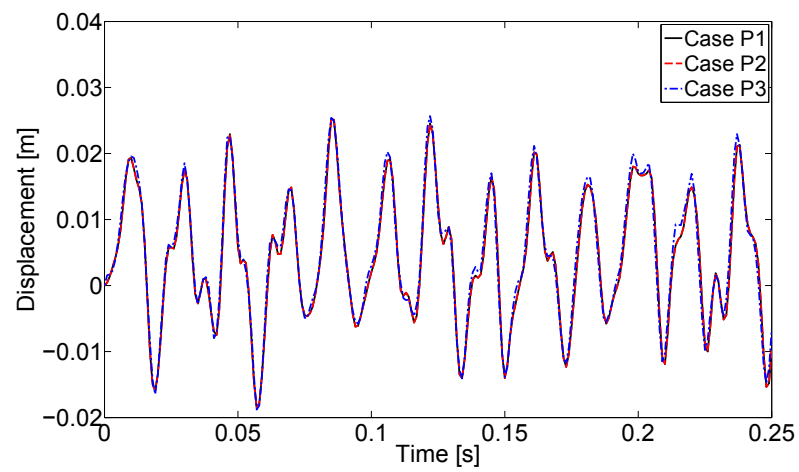

(b) Node 2 (bottom surface, root leading edge).

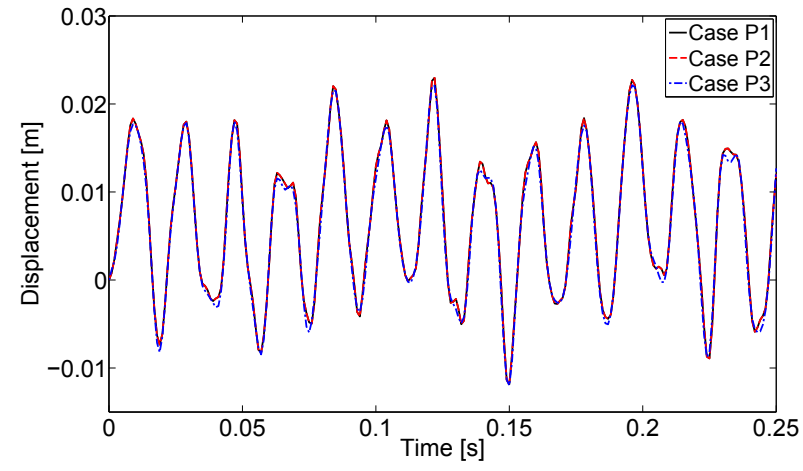

(d) Node 458 (bottom surface, root trailing edge).

Figure 14. Time-history of $z$ displacements at four selected nodes for cases P1 - P3.

Plots of the $z$-direction displacements from cases P1-P3 are given in Fig. 14 for four selected nodes. Examining the results, the first observation to be made is that, as expected, case P1 matches case P2 nearly exactly indicating that the partitioned approach is mathematically equivalent to the monolithic system approach. Comparing case P3 to case P2, it can be seen that the control surface structural ROM shows only minor discrepancies with respect to the full-order model, and thus the accuracy of this ROM is sufficient for the purposes of this study. As shown in Fig. 14(a), the fuselage node oscillates at approximately $10 \mathrm{~Hz}$ which was the targeted frequency for the uncoupled single DOF spring/mass system. For the properties chosen for this case, the loads imparted by the control surface on the fuselage do not cause the fuselage to deviate significantly from a single DOF oscillation response. Subsequent studies will further explore the extent to which the control surface motion impacts the fuselage response under different types of excitations. Finally, it should be noted that only one iteration was required for convergence at all time steps for cases P2 and $\mathrm{P} 3$. This is due to the fact that the $R$ is a linear function of $x_{r}^{(n+1)}$ for the configuration under investigation. 
Thus, once $R$ is evaluated at the two initial guesses generated in step 1 of Table 2, the slope of $R$ is known and the converged value of $x_{r}^{(n+1)}$ can be obtained in the first iteration of the secant method. However, the methodology is intentionally formulated to be general such that the problem need not be linear. If the problem is in fact linear, then the linear solution is recovered in the minimum number of iterations.

\section{VI.B. Impact of Control Surface Inertia on Fuselage $z$ Direction Response}

With the control surface ROM and partitioned solution methodology validated, the framework is now used to assess the impact of control surface inertial loads. In order to assess these effects, the coupled fuselagecontrol surface formulation described previously is used to perform simulations of the system depicted in Fig. 10 in which control surface inertial effects are both present and absent. For the case in which control surface inertial effects are present, the coupled formulation described previously, which includes control surface structural dynamics, is employed. For the case in which control surface inertial effects are absent, the coupled formulation is modified such that the solution for the control surface deformation is reduced to the solution of a static problem subjected to thermal and aerodynamic loads with the constraint motion imposed instantaneously. Elimination of control surface inertial effects is achieved by modifying Eq. (7b) to include only static terms such that it becomes

$$
K_{u r}^{*} x_{r}+K_{u u}^{*} x_{u}=F_{u}^{H}+F_{u}^{A} .
$$

In order to maintain consistency with the dynamic control surface solution, Eq. (34) is solved in a manner analogous to that derived in Eqs. (8) - (17). Therefore, for the static control surface case, the equations governing the control surface modal coordinates are reduced to

$$
k_{u u}^{*}(T) d(t)=f_{u}(t, T) .
$$

The net external force exerted by the control surface relative to the fuselage, $F_{r}^{W / B}$, is again calculated using Eq. (25) for the static control surface case. However, for the static control surface case, $x_{u}^{E}$ will not include elastic deformation due to control surface inertia, and will thus allow for assessing the impact of control surface inertia on the loads that the control surface exerts on the fuselage.

Before performing simulations, $M_{f}$ is adjusted such that the fuselage weight is exactly balanced by the lift force produced by the control surface at $M_{\infty}=6.5, \alpha_{\text {net }}=2^{\circ}, h=35 \mathrm{~km}$. To calculate this lift force, the control surface is first heated for $400 \mathrm{~s}$ at these flight conditions in order to obtain a representative temperature distribution. It is then fixed at its attachment point and brought to elastic equilibrium under thermal loads and aerodynamic loads. Once elastic steady state is obtained, $F_{r}^{W / B}$ is calculated in order to obtain the lift force that is generated by the control surface and transferred the fuselage. Additionally, the spring stiffness associated with the fuselage is set such that the fuselage natural frequency is $10 \mathrm{~Hz}$ in the absence of control surface inertia.

All simulations in this section begin by heating the control surface for $400 \mathrm{~s}$ at $M_{\infty}=6.5, \alpha_{\text {net }}=2^{\circ}$, $h=35 \mathrm{~km}$ and bringing it to elastic equilibrium under thermal and aerodynamic loads. As the weight of the fuselage is equal to the lift produced by the control surface under these conditions, the fuselage will be at its equilibrium position at the beginning of the simulation. The simulation is then started at these flight conditions by time-marching the partitioned control surface-fuselage formulation forward in the manner described previously. For the results presented, the time step sizes are chosen as follows: $\Delta t_{A E}=\Delta t_{H T}=$ $0.001 \mathrm{~s}$ and $\Delta t_{A T}=0.1 \mathrm{~s}$. Though the thermal and aerodynamic loads will change over time as the simulation progresses, the impact of these loads on the response of the system is minimal as they change on a slow time scale. Thus, it is necessary to perturb the equilibrium conditions such that structural dynamic oscillations are induced into the system so that the impact of inertial effects can be examined. The method used here to perturb the equilibrium is to apply a change in flow direction for a short period of time, which corresponds to a change in control surface angle of attack, $\alpha_{\text {net }}$. The flow direction is instantaneously incremented at time $t_{i}$ by an amount $\Delta \alpha_{\text {net }}$, held it at this value for a time duration $\Delta t_{\alpha}$, and then decremented back to the original value of $\alpha_{\text {net }}$ at time $t_{d}=t_{i}+\Delta t_{\alpha}$. The results presented in this paper use $\Delta \alpha_{\text {net }}=8^{\circ}$ which corresponds to increasing $\alpha_{\text {net }}$ from $2^{\circ}$ to $10^{\circ}$ at $t_{i}$, holding at $10^{\circ}$ for a time duration of $\Delta t_{\alpha}$, and then decrementing back to $2^{\circ}$ at $t_{d}$. The values of $\Delta t_{\alpha}$ that are used in the simulations are $0.03 \mathrm{~s}$ and $0.1 \mathrm{~s}$. Note that for the results presented, $\Delta \alpha_{\text {net }}$ is applied at $0.01 \mathrm{~s}$ into the transient $\left(t_{i}=0.01 \mathrm{~s}\right)$.

The displacements of the mass representing the fuselage are shown in Fig. 15 for a one second time-history where "CS Dynamic" refers to the case which utilizes the structural dynamic solution to obtain the control 
surface response at each aeroelastic time step, while "CS Static" refers to the case which utilizes the static solution to obtain the control surface response at each aeroelastic time step. Figure 15(a) shows results for the case of $\Delta t_{\alpha}=0.03 \mathrm{~s}$, while Fig. 15(b) shows results for the case of $\Delta t_{\alpha}=0.1 \mathrm{~s}$. For $\Delta t_{\alpha}=0.03 \mathrm{~s}$, $\alpha_{\text {net }}$ is decremented back from $10^{\circ}$ to $2^{\circ}$ just before the fuselage reaches the peak of its response. However, for $\Delta t_{\alpha}=0.1 \mathrm{~s}, \alpha_{\text {net }}$ is not decremented back from $10^{\circ}$ to $2^{\circ}$ until the fuselage has approximately returned its equilibrium position. Therefore, comparing Fig. 15(a) to Fig. 15(b), it can be seen that the fuselage undergoes significantly higher amplitude oscillations for the case of $\Delta t_{\alpha}=0.03 \mathrm{~s}$ than for the case of $\Delta t_{\alpha}=0.1 \mathrm{~s}$. Comparing the "CS Dynamic" and "CS Static" cases in Fig. 15(a), one can observe a difference in frequency of oscillation resulting from the inclusion of control surface inertia. Recall that the mass and stiffness properties associated with the fuselage were set such that its natural frequency would be $10 \mathrm{~Hz}$ in the absence of control surface inertia. Examining the "CS Static" case in Fig. 15(a), this $10 \mathrm{~Hz}$ natural frequency is observed. However, by including control surface inertia, the frequency of fuselage oscillation is reduced as shown by the "CS Dynamic" case in Fig. 15(a). Note that for the case of $\Delta t_{\alpha}=0.03 \mathrm{~s}$, control surface inertia has only a minor effect on the amplitude of fuselage oscillation for the "CS Dynamic" case. Examining Fig. 15(b) it can be seen that when $\Delta t_{\alpha}$ is increased to $0.1 \mathrm{~s}$, control surface inertia has a more prominent effect on the amplitude of the fuselage response. This is due to the fact that $\alpha_{\text {net }}$ is decremented back to $2^{\circ}$ approximately at the same time that the fuselage reaches its equilibrium position. Thus for the "CS Static" case, the amplitude of fuselage oscillation is reduced significantly. The result is that the forces acting on the fuselage due to its own inertia and stiffness are smaller compared to the control surface inertial loads beyond $t_{d}$. Therefore, control surface inertia has a more prominent effect on fuselage amplitude of oscillation beyond $t_{d}$ for this case.

In order to examine the frequency content of the fuselage response, the fast Fourier transform (FFT) is performed on the fuselage response for the time-history beginning at $t_{d}$. Because the FFT assumes that the input sequence is a power of two in length, ${ }^{35}$ the FFT is performed on a signal in the time range 0.041 $\mathrm{s} \leq t \leq 1.064 \mathrm{~s}$ for $\Delta t_{\alpha}=0.03 \mathrm{~s}$ and in the range $0.111 \mathrm{~s} \leq t \leq 1.134 \mathrm{~s}$ for $\Delta t_{\alpha}=0.1 \mathrm{~s}$. Therefore, the output signal is of length 1,024 for both values of $\Delta t_{\alpha}$. The single-sided amplitude spectrums of the fuselage displacements are given in Fig. 16(a) and Fig. 16(b) for the cases of $\Delta t_{\alpha}=0.03 \mathrm{~s}$ and $\Delta t_{\alpha}=0.1 \mathrm{~s}$, respectively. For both values of $\Delta t_{\alpha}$, the first peak at nonzero frequency occurs at approximately $8.79 \mathrm{~Hz}$ for the "CS Dynamic" case and at approximately $9.77 \mathrm{~Hz}$ for the "CS Static" case, indicating that exclusion of control surface inertia results in an $11 \%$ overprediction in the lowest-frequency fuselage oscillation component. Based on the frequency of oscillation, it can be concluded that this mode is associated with the single DOF mass-spring fuselage system. In addition to this fuselage mode, results from the "CS Dynamic" case show higher frequency oscillation components associated with control surface modes. For both values of $\Delta t_{\alpha}$, the second and third major peaks occur at approximately $26.4 \mathrm{~Hz}$ and $54.7 \mathrm{~Hz}$, respectively. In order to determine which control surface modes these frequencies correspond to, the first ten control surface natural frequencies are computed at the actual thermal state of the structure which is obtained by heating the control surface for $400 \mathrm{~s}$ at the previously described flight conditions. These first ten natural frequencies are given in Table 7. Based on the frequencies shown in the table, it can be concluded that the second major peaks for the "CS Dynamic" cases in both Figs. 16(a) and 16(b) correspond to the first control surface mode, while the third major peaks correspond to either the third or fourth control surface mode. It should be noted that a previous work ${ }^{15}$ described a mode switching phenomenon that was found to occur between control surface modes three and four as the structure is heated. This phenomenon is likely due to the fact that these modes are close in frequency. Though there are three noticeable peaks present in the FFT results of the fuselage displacements, the lowest frequency mode clearly contributes most significantly to the response for both values of $\Delta t_{\alpha}$.

Plots of the control surface $z$ displacements at node 247 (located on the bottom surface at the mid-chord of the tip) are shown in Figs. 17(a) and 17(b) for $\Delta t_{\alpha}=0.03 \mathrm{~s}$ and $\Delta t_{\alpha}=0.1 \mathrm{~s}$, respectively. For the "CS Static" case in both figures, there is an instantaneous jump in displacement at $t_{i}=0.01 \mathrm{~s}$. This is due to the increase in aerodynamic pressure loads on the structure induced by the increased angle of attack. Because the control surface response is calculated using a static solution for the "CS Static" cases, the increase in displacement is instantaneous for these cases. Between $t_{i}$ and $t_{d}$ for the static cases, the control surface motion approximately follows the constraint motion imposed by the fuselage. At $t_{d}$ when $\alpha_{\text {net }}$ is decremented back to $2^{\circ}$, the static control surface displacement instaneously drops due to the decrease in aerodynamic loads. Beyond $t_{d}$, the control surface motion in the "CS Static" case is approximately the same as the fuselage motion with an offset due to static deformation under thermal and aerodynamic loads. Note that 


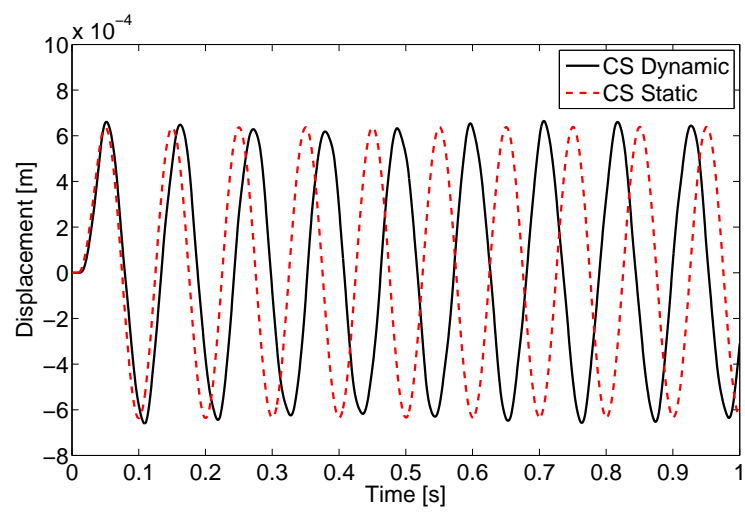

(a) $\Delta t_{\alpha}=0.03 \mathrm{~s}$.

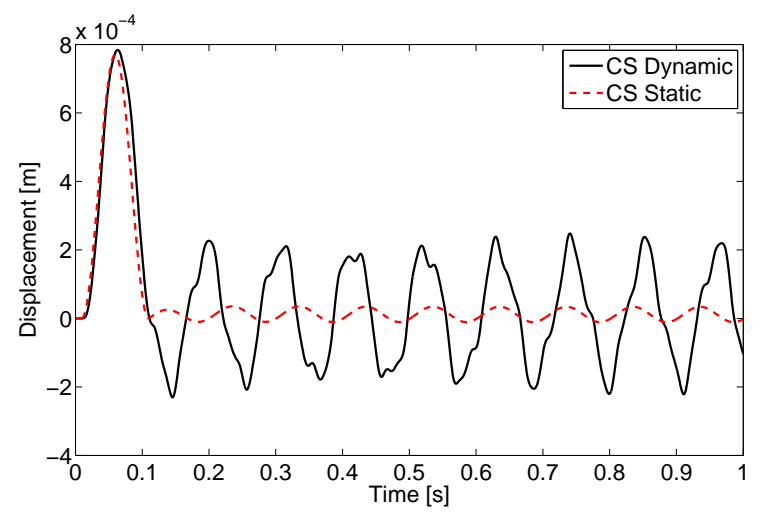

(b) $\Delta t_{\alpha}=0.1 \mathrm{~s}$.

Figure 15. Fuselage displacements for two values of $\Delta t_{\alpha}$ with and without control surface inertial effects.

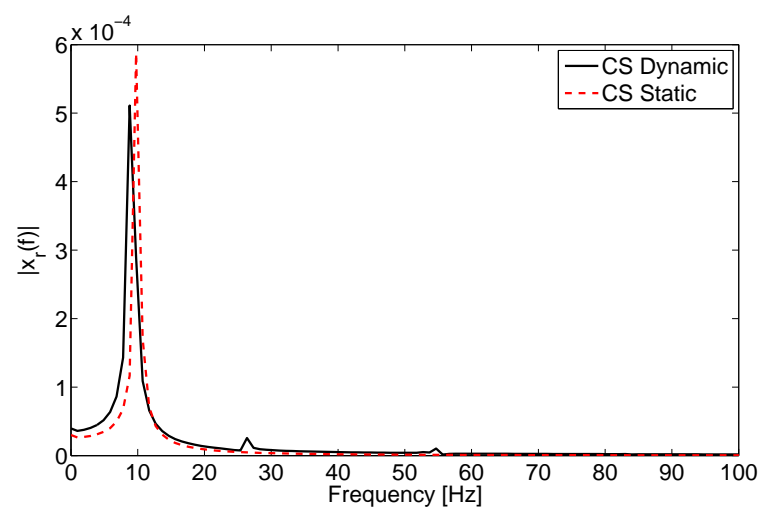

(a) $\Delta t_{\alpha}=0.03 \mathrm{~s}$.

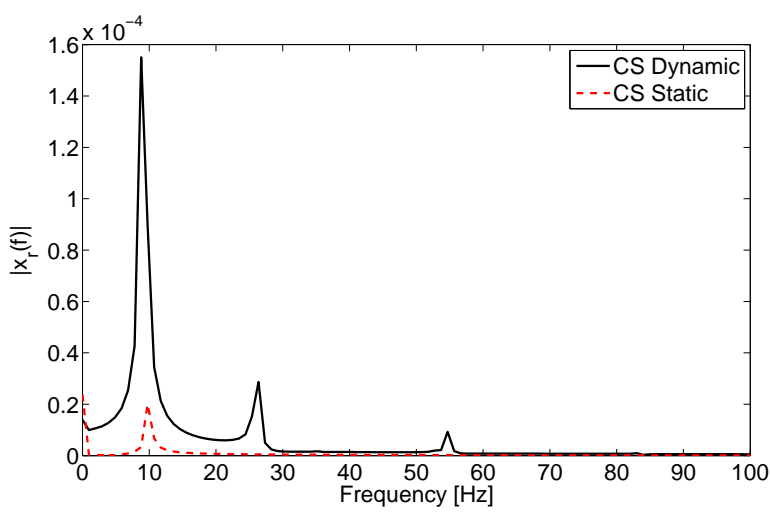

(b) $\Delta t_{\alpha}=0.1 \mathrm{~s}$.

Figure 16. FFT of fuselage response for two values of $\Delta t_{\alpha}$ with and without control surface inertial effects.

Table 7. First ten control surface natural frequencies evaluated thermal state obtained by heating structure for $400 \mathrm{~s}$ at $M_{\infty}=6.5, \alpha_{\text {net }}=2^{\circ}$, and $h=35 \mathrm{~km}$.

\begin{tabular}{cc}
\hline \hline Mode Number & Natural Frequency $[\mathrm{Hz}]$ \\
\hline 1 & 25.1 \\
2 & 35.6 \\
3 & 53.2 \\
4 & 54.3 \\
5 & 86.1 \\
6 & 98.1 \\
7 & 130 \\
8 & 144 \\
9 & 163 \\
10 & 183 \\
\hline \hline
\end{tabular}


while the thermal and aerodynamic loads do change over time in the static case, their effect on control surface deformation is essentially a static effect as these loads change on a slow time scale. Comparing the "CS Dynamic" cases to the "CS Static" cases, it can be observed that sustained structural dynamic oscillations are induced in the control surface which lead to higher displacement levels than would be predicted if only a static control surface solution was used.

The FFT's of the control surface response at node 247 are taken for the same time periods as used in generating Figs. 16(a) and 16(b). The single-sided amplitude spectrums for the two values of $\Delta t_{\alpha}$ are given in Figs. 18(a) and 18(b). For both values of $\Delta t_{\alpha}$, the first major peak at nonzero frequency occurs at approximately $8.79 \mathrm{~Hz}$ in the "CS Dynamic" case and at approximately $9.77 \mathrm{~Hz}$ in the "CS Static" case. For the "CS Dynamic" case, the second major peak occurs at approximately $26.4 \mathrm{~Hz}$ for both values of $\Delta t_{\alpha}$. The frequencies of the first two control surface peaks are the same as those for the first two fuselage peaks. While these first two peaks contribute most significantly to the control surface response for the "CS Dynamic" case, there exist three additional smaller peaks for both values of $\Delta t_{\alpha}$ which occur at approximately 35.2 $\mathrm{Hz}, 54.7 \mathrm{~Hz}$, and $83.0 \mathrm{~Hz}$. Referring to Table 7, these three peaks correspond roughly with control surface modes two, three/four, and five, respectively. Comparing the control surface FFT results with those of the fuselage, it can be seen that while control surface modes $1-5$ are all excited at least to a small extent in the control surface response, only control surface modes one and three/four appear to have an effect on the fuselage response. However, as was the case for the fuselage, the two lowest frequency modes contribute most significantly to the control surface response.

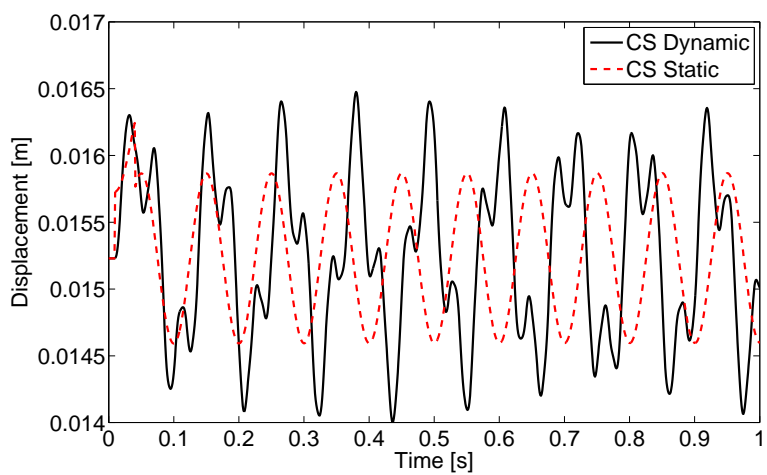

(a) $\Delta t_{\alpha}=0.03 \mathrm{~s}$.

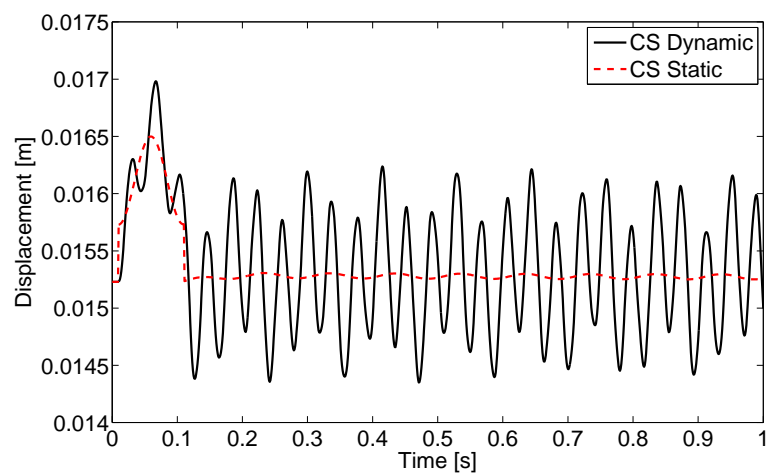

(b) $\Delta t_{\alpha}=0.1 \mathrm{~s}$.

Figure 17. Control surface $z$ displacements at node 247 (bottom surface, tip, mid-chord) for two values of $\Delta t_{\alpha}$ with and without control surface inertial effects.

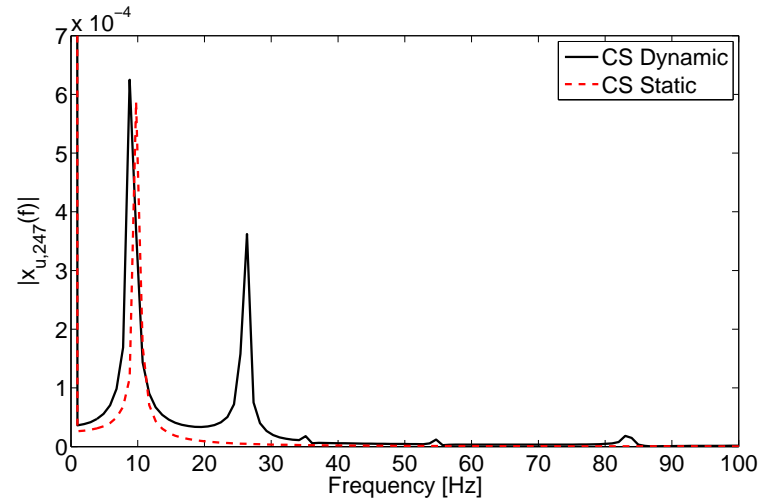

(a) $\Delta t_{\alpha}=0.03 \mathrm{~s}$.

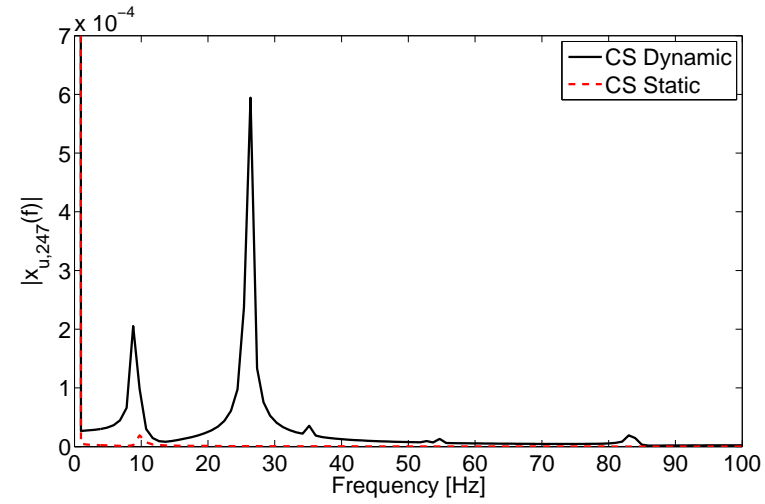

(b) $\Delta t_{\alpha}=0.1 \mathrm{~s}$.

Figure 18. FFT of control surface response at node 247 (bottom surface, tip, mid-chord) for two values of $\Delta t_{\alpha}$ with and without control surface inertial effects.

The loads exerted by the control surface on the fuselage, $F_{r}^{W / B}$, are shown in Fig. 19 for the two values of $\Delta t_{\alpha}$. As expected, in the "CS Static" cases, $F_{r}^{W / B}$ is approximately constant with an instantaneous increase 
at $t_{i}$ and instantaneous decrease at $t_{d}$ due to the change in $\alpha_{\text {net }}$. Examining the "CS Dynamic" results, $F_{r}^{W / B}$ is shown to oscillate approximately about the static value due to the control surface inertial effects. As shown by the fuselage displacements in Fig. 15, the extent to which the fuselage responds to these highfrequency oscillations in $F_{r}^{W / B}$ depends upon the inertia and stiffness of the fuselage itself. Based on the results presented for this time history, the maximum absolute ratio of $F_{r}^{W / B}$ for the dynamic case to that for the static case is seven for $\Delta t_{\alpha}=0.03 \mathrm{~s}$ and eight for $\Delta t_{\alpha}=0.1 \mathrm{~s}$. These results indicate that control surface inertia can have a noticeable effect on fuselage loads. However, the extent to which control surface inertial loads impact the fuselage response is dependent on the fuselage inertia as well as the frequency content of the control surface inertia loads.

Plots of the FFT of the $F_{r}^{W / B}$ time-history for the two values of $\Delta t_{\alpha}$ are given in Figs. 20(a) and 20(b). In both plots, noticeable peaks occur for the "CS Dynamic" case at approximately 8.79 Hz, 26.4 Hz, 52.7 $\mathrm{Hz}, 54.7 \mathrm{~Hz}, 84.0 \mathrm{~Hz}, 121 \mathrm{~Hz}, 132 \mathrm{~Hz}, 146 \mathrm{~Hz}$, and $160 \mathrm{~Hz}$. While the modes at these frequencies each contribute to $F_{r}^{W / B}$ at least to a small extent, the modes corresponding to the first four of these frequencies contribute most significantly. Referring to Table 7, these four frequencies roughly correspond to fuselage mode 1 , control surface mode 1 , control surface mode 3 , and control surface mode 4 . The most dominant contribution to $F_{r}^{W / B}$ comes from the $54.7 \mathrm{~Hz}$ mode for both values of $\Delta t_{\alpha}$. However, it is interesting to note that the $54.7 \mathrm{~Hz}$ mode is not the most dominant mode in either the fuselage response or control surface response.

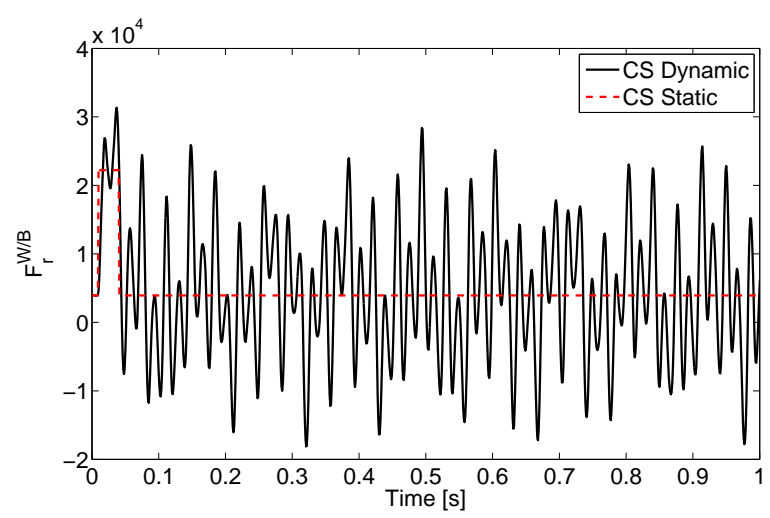

(a) $\Delta t_{\alpha}=0.03 \mathrm{~s}$

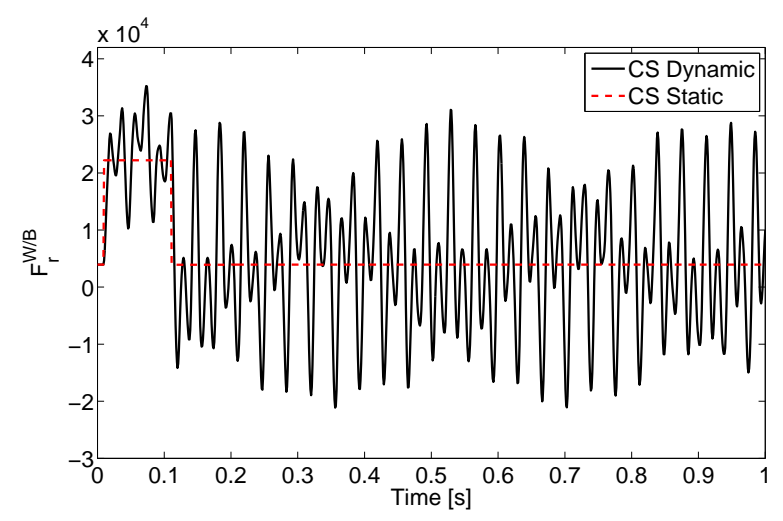

(b) $\Delta t_{\alpha}=0.1 \mathrm{~s}$.

Figure 19. $F_{r}^{W / B}$ for two values of $\Delta t_{\alpha}$ with and without control surface inertial effects.

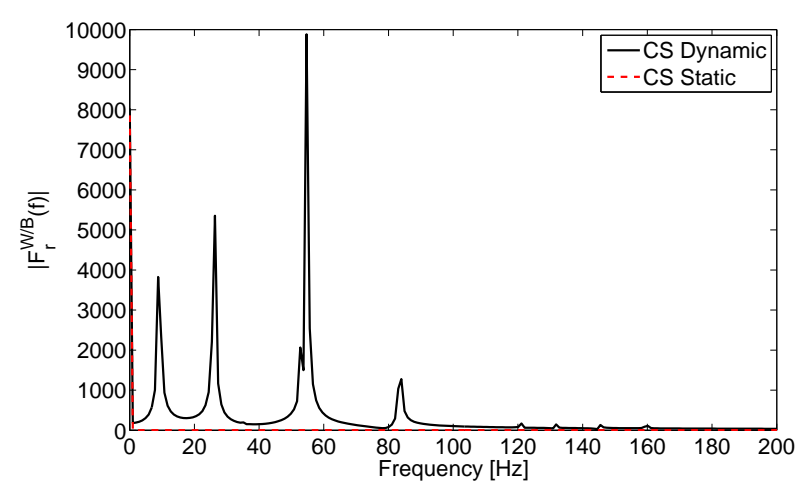

(a) $\Delta t_{\alpha}=0.03 \mathrm{~s}$.

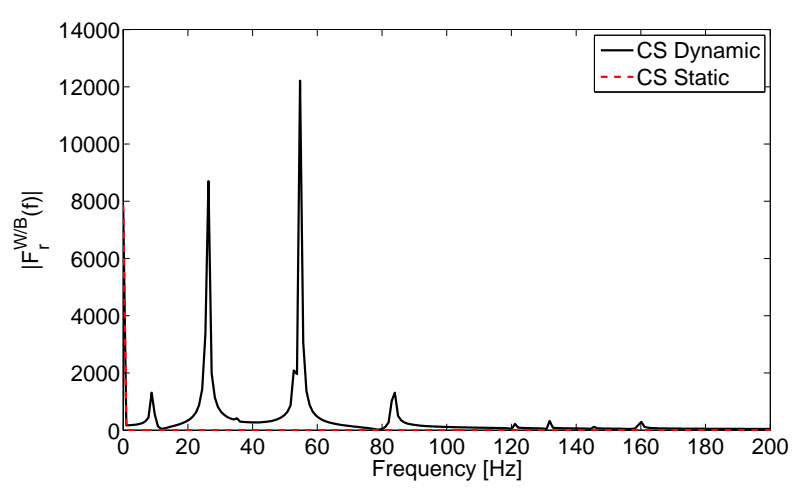

(b) $\Delta t_{\alpha}=0.1 \mathrm{~s}$.

Figure 20. FFT of $F_{r}^{W / B}$ response for two values of $\Delta t_{\alpha}$ with and without control surface inertial effects. 


\section{VI.C. Impact of Control Surface Inertia Under Commanded Change in Deflection Angle}

The next aspect of this study involves investigation of the impact of control surface inertial loads under a commanded change in control surface deflection angle imposed via the control system. The importance of understanding such control surface-fuselage inertial coupling has been discussed in a recent paper ${ }^{9}$ which referred to this coupling as the "tail-wags-dog" effect. The discussion in Ref. 9 highlights the fact that this effect typically results in a complex-conjugate pair of zeros in the elevator-to-pitch rate transfer function and can affect the speed of response of the system.

As a step toward assessing the extent to which the overall vehicle pitch response is affected by control surface inertia under commanded changes in deflection angle, a control input corresponding to control surface deflection angle is incorporated into the aerothermoelastic ROM framework. Note that all simulations conducted in this section do not include the fuselage mass depicted in Fig. 10, and consist of enforced control surface motion due to rotation about the hinge line. In order to capture the relationship between the input command from the controller and the resulting output rotation applied to the control surface, actuator dynamics are incorporated into the control surface model. The equation relating the input command, $\delta_{\mathrm{cmd}}$, to the output control surface deflection angle, $\delta$, is given by ${ }^{37}$

$$
\ddot{\delta}=-2 \zeta_{\delta} \omega_{\delta} \dot{\delta}-\omega_{\delta}^{2} \delta+\omega_{\delta} \delta_{\mathrm{cmd}},
$$

where $\zeta_{\delta}=1$ and $\omega_{\delta}=20$. A schematic illustrating the geometry associated with the control surface deflection angle is given in Fig. 21, where $x_{B}$ and $z_{B}$ represent the body-fixed axis system. As shown in Fig. 21, $\delta$ is taken to be positive leading edge up. Note that the net control surface angle of attack, $\alpha_{\text {net }}$, is given by

$$
\alpha_{\text {net }}=\alpha+\delta
$$

where $\alpha$ is the vehicle angle of attack.

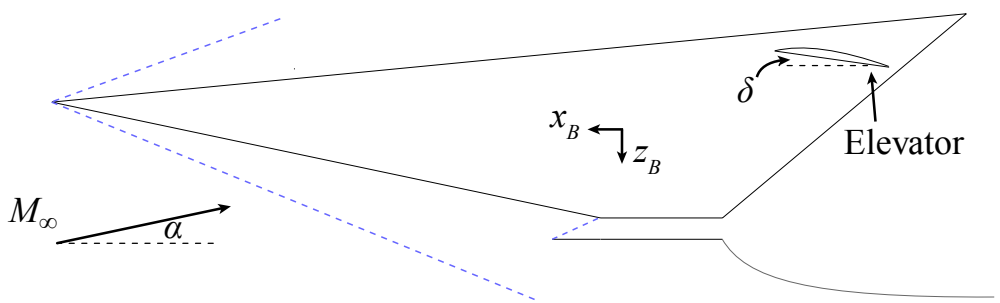

Figure 21. Schematic illustrating geometry associated with control surface deflection angle and vehicle angle of attack.

\section{VI.C.1. Commanded Step Increase in Control Surface Deflection Angle}

For the first example case of a change in control surface deflection angle, the input command is taken to be a step change in deflection angle that is applied at $0.01 \mathrm{~s}$ into the simulation. The input step command corresponds to an instantenous increase in the desired value of $\delta$ from $0^{\circ}$ to $3.9^{\circ}$. Because the simulations are carried out for $\alpha=0.1^{\circ}$, the final value of $\delta$ is chosen to be $3.9^{\circ}$ such that the total flow angle with respect to the control surface remains within the bounds on $\alpha_{\text {net }}$ given in Table 3. The functional form of the the input command is given by

$$
\delta_{\mathrm{cmd}}(t)=20 H(t-\tau)\left[3.9^{\circ} \frac{\pi}{180^{\circ}}\right]
$$

where $H(t-\tau)$ is the Heaviside step function and $\tau$ is the time delay which is taken to be $0.01 \mathrm{~s}$ in this case. A plot showing $\delta(t)$ based on the input command of Eq. (38) is given in Fig. 22, where $\delta$ has been converted from radians to degrees for visualization purposes.

Simulations are carried out using the input command described above at $M_{\infty}=6.5$ and $h=35 \mathrm{~km}$. The time step sizes are chosen as follows: $\Delta t_{A E}=\Delta t_{H T}=0.001 \mathrm{~s}$ and $\Delta t_{A T}=0.1 \mathrm{~s}$. As was done in the previous section, the control surface is first heated for $400 \mathrm{~s}$ at this Mach number and altitude at $\alpha=0.1^{\circ}$ and $\delta=0^{\circ}$. Using the temperature distribution obtained at the end of the $400 \mathrm{~s}$ period, the control surface is brought to aerothermoelastic equilibrium, and the simulation is then started from initial time. The vehicle angle of attack, $\alpha$, is held at $0.1^{\circ}$ throughout the simulation, and $\delta$ is given by the time-history shown in Fig. 22. The 


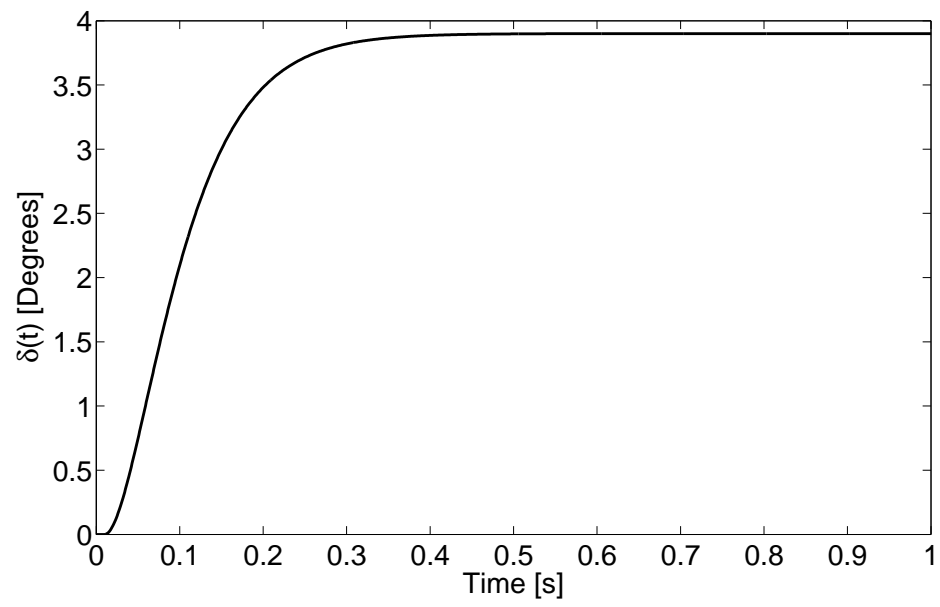

Figure 22. Time-history of applied control surface deflection angle, $\delta(t)$.

entries of $x_{u}$ corresponding to the $z$ displacements of node 37 (located at the bottom surface, tip, leading edge) and node 475 (located on the bottom surface, trailing edge) are plotted as a function of time in Fig. 23. Note that $x_{u}$ includes both the constraint motion due to the change in control surface deflection angle, $x_{u}^{C}$, as well as the elastic motion relative to the constraint motion, $x_{u}^{E}$, as given in Eq. (8). As in the previous section, the "CS Dynamic" case includes control surface structural dynamics, while the "CS Static" case uses only a static solution to obtain the control surface response at each time step. Comparing the two cases for the time range $0.5 \mathrm{~s} \leq t \leq 2.0 \mathrm{~s}$, it is found that exclusion of control surface inertia results in a maximum absolute percent error of $5.6 \%$ for the node $37 z$ displacements and $2.3 \%$ for the node $475 z$ displacements.

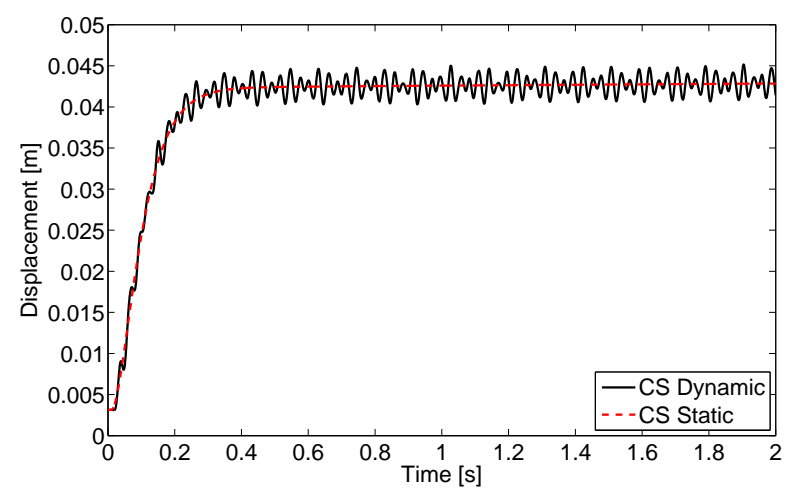

(a) Node 37 (bottom surface, tip, leading edge).

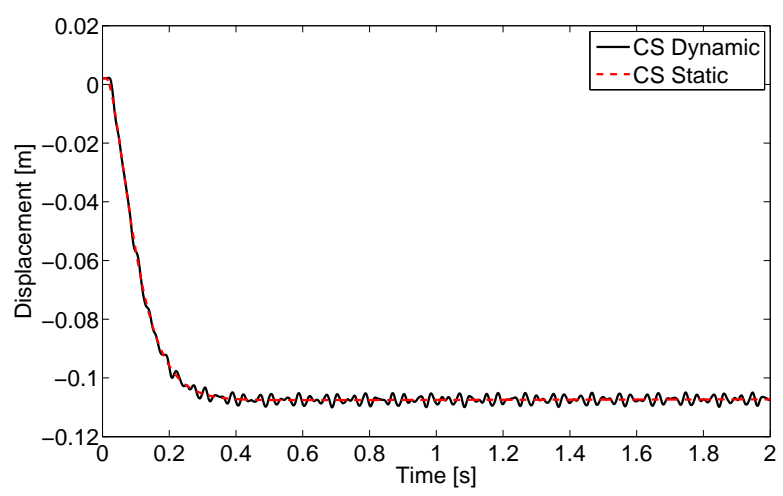

(b) Node 475 (bottom surface, tip, trailing edge).

Figure 23. Control surface total displacements, $x_{u}$, in $z$ direction under commanded change in deflection angle with and without control surface inertial effects for two selected nodes.

In order to remove the effect of the constraint displacements due to the control surface deflection angle, the elastic displacements relative to the constraint motion, $x_{u}^{E}$, are also analyzed. The entries of $x_{u}^{E}$ corresponding to the $z$ displacements of nodes 37 and 475 are plotted in Fig. 24. By isolating the elastic component of the displacements, the effect of control surface inertia on its response can be seen more clearly. For the "CS Static" case, there is a small monotonic increase in elastic displacements over time due to increased steady aerodynamic loads and thermal loads as $\delta$ is increased. Examining the "CS Dynamic" results, it is observed that the change in control surface deflection angle induces noticeable structural dynamic oscillations about the statically deformed state. Comparing the "CS Dynamic" case with the "CS Static" case for the time range $0.5 \mathrm{~s} \leq t \leq 2.0 \mathrm{~s}$, inclusion of control surface inertia results in elastic displacements of up to 1.7 times and 1.9 times the corresponding static displacements for node 37 and 475, respectively. The maximum absolute elastic displacements in the "CS Dynamic" case for the complete time histories shown in Figs. 24(b) 
and 24(a) are $0.0062 \mathrm{~m}$ (0.24 in) for node 37 , and $0.0053 \mathrm{~m}$ (0.21 in) for node 475.

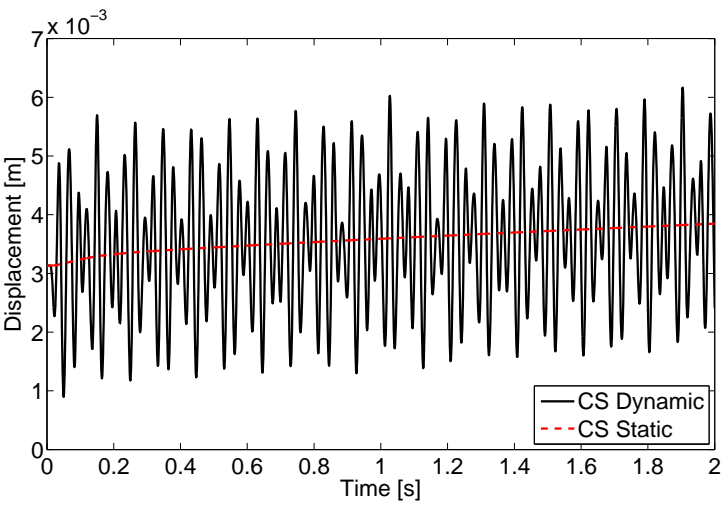

(a) Node 37 (bottom surface, tip, leading edge).

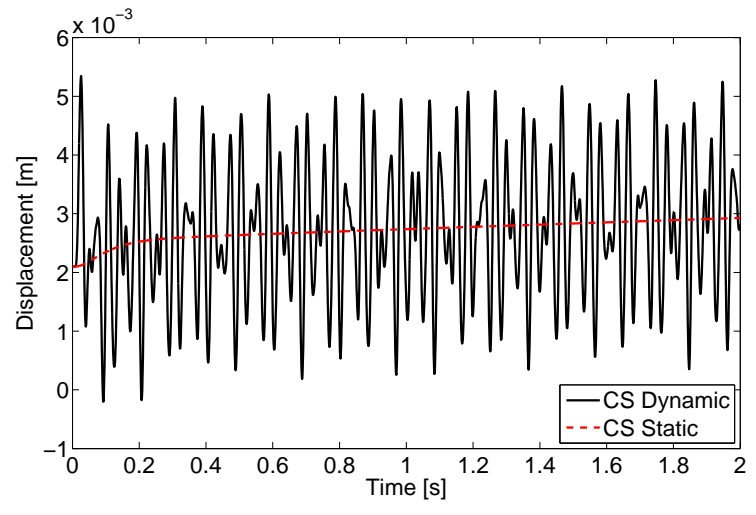

(b) Node 475 (bottom surface, tip, trailing edge).

Figure 24. Control surface elastic displacements, $x_{u}^{E}$, in $z$ direction under commanded change in deflection angle with and without control surface inertial effects.

While examination of the displacement time-histories of the control surface provides insight into the response of the system, one of the main goals of this study is to assess the impact of control surface inertia on the overall dynamics of the HSV. As discussed at the beginning of the current section, an important result of control surface-fuselage inertial coupling is that the response of the vehicle pitch rate to changes in elevator deflection angle can be adversely affected. As a step toward quantifying such effects, the pitching moments exerted by the control surface on the fuselage at the attachment point, $M_{y}^{W / B}$, are examined for this case. The time-histories of $M_{y}^{W / B}$ for the static and dynamic control surface cases are given in Fig. 25(a) and Fig. 25(b), respectively. Note that a positive value of $M_{y}^{W / B}$ corresponds to a nose-up pitching moment due to the orientation of the control surface coordinate system. For the static control surface case, as $\delta$ approaches the commanded value of the deflection angle, $M_{y}^{W / B}$ asymptotically approaches a higher value. However, for the dynamic control surface case, the increase in $M_{y}^{W / B}$ over time is essentially indiscernible due to oscillations resulting from control surface inertia. For the time range $0.5 \mathrm{~s} \leq t \leq 2.0 \mathrm{~s}$, the maximum absolute ratio of $M_{y}^{W / B}$ from the dynamic control surface case to that from the static control surface case is 127 , indicating that control surface inertia can significantly impact the pitching moment from the control surface under commanded changes in control surface deflection angle. Therefore, exclusion of control surface inertia may result in errors in vehicle pitch response prediction.

For the static control surface case, $M_{y}^{W / B}$ initially drops when the commanded change in $\delta$ is first applied at $0.01 \mathrm{~s}$. This is due to the velocity induced as the control surface begins to rotate about the hinge line. This velocity leads to unsteady aerodynamic pressure loads which counteract the positive moment caused by the steady pressure loads. Once $\delta$ reaches a certain deflection angle, the negative moment caused by the unsteady aerodynamic pressure loads is balanced out by the positive moment due to the steady pressure loads, and $M_{y}^{W / B}$ begins to increase. Additionally, small discontinuities in $M_{y}^{W / B}$ are observed for the static control surface case at $0.1 \mathrm{~s}, 0.2 \mathrm{~s}$, and $0.3 \mathrm{~s}$. These discontinuities are a result of the updating of the skin friction which occurs along with the updating of the thermal boundary conditions in intervals of $0.1 \mathrm{~s}$. The increase in control surface deflection angle leads to increased dynamic pressure and thus elevated skin friction. Because the skin friction on the bottom surface is increased by a greater amount than that on the top surface due to the positive control surface angle of attack, there is an instantaneous decrease in $M_{y}^{W / B}$ each time the skin friction is updated up to approximately $0.3 \mathrm{~s}$.

To assess the frequency content of the $M_{y}^{W / B}$ time-history, the FFT of the dynamic control surface case is taken for the time range $0.5 \mathrm{~s} \leq t \leq 1.523 \mathrm{~s}$. The resulting single-sided amplitude spectrum is given in Fig. 26. As shown in the figure, there exist three main control surface structural dynamic modes which contribute to the $M_{y}^{W / B}$ response. The frequencies of the first three peaks are approximately $25.4 \mathrm{~Hz}, 35.2$ $\mathrm{Hz}$, and $52.7 \mathrm{~Hz}$. In order to identify which control surface modes correspond to these three peaks, the first four control surface free vibration mode shapes and frequencies are computed at the corresponding thermal state. This thermal state is obtained by heating the structure for $400 \mathrm{~s}$ at the following flight conditions: $M_{\infty}=6.5, \alpha=0.1^{\circ}, \delta=0^{\circ}$, and $h=35 \mathrm{~km}$. The resulting mode shapes and their frequencies are given 
in Fig. 27. Based on the frequencies given Fig. 27, the first, second, and third peaks in Fig. 26, roughly correspond with control surface mode one, mode two, and mode three or four, respectively. The dominant peak in Fig. 26 is the second peak, which corresponds roughly with control surface mode two. Examining the mode shape of control surface mode two, it is clear that this mode has a significant torsion component about the attachment location, and one would therefore expect that this mode would contribute most significantly to $M_{y}^{W / B}$ dynamic response. Thus, if one were designing a control system aimed at stabilizing the HSV pitching moment, the second control surface mode would be the most critical mode to control.

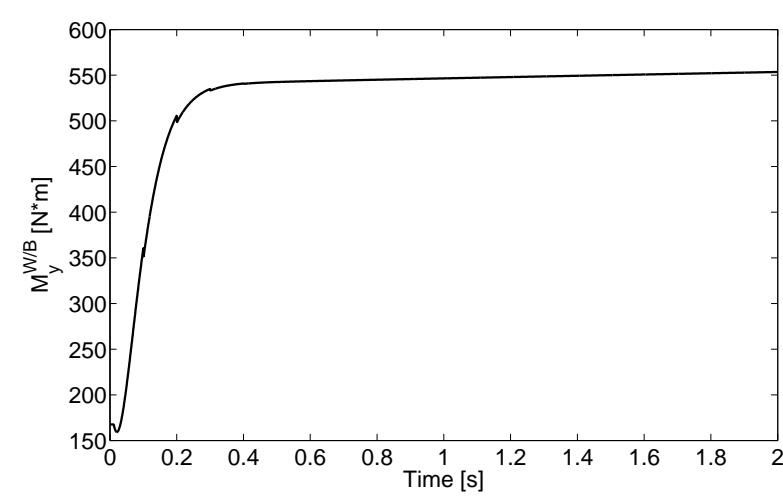

(a) Static control surface case.

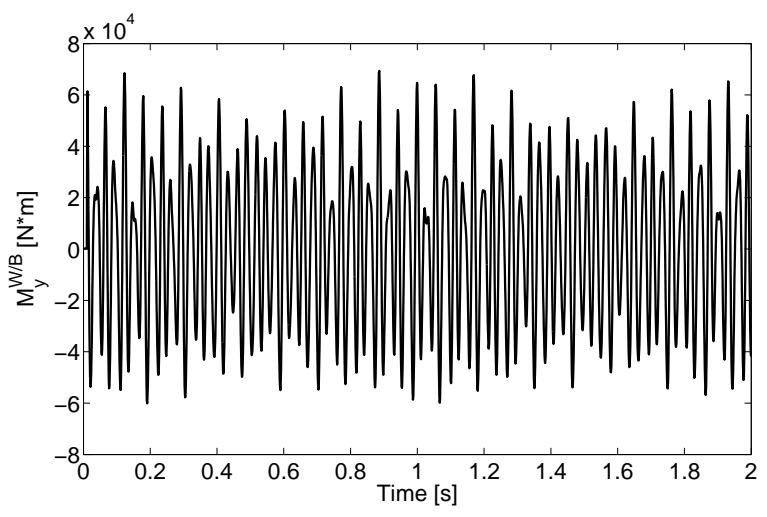

(b) Dynamic control surface case.

Figure 25. Time-domain response of $M_{y}^{W / B}$ for both static and dynamic control surface cases.

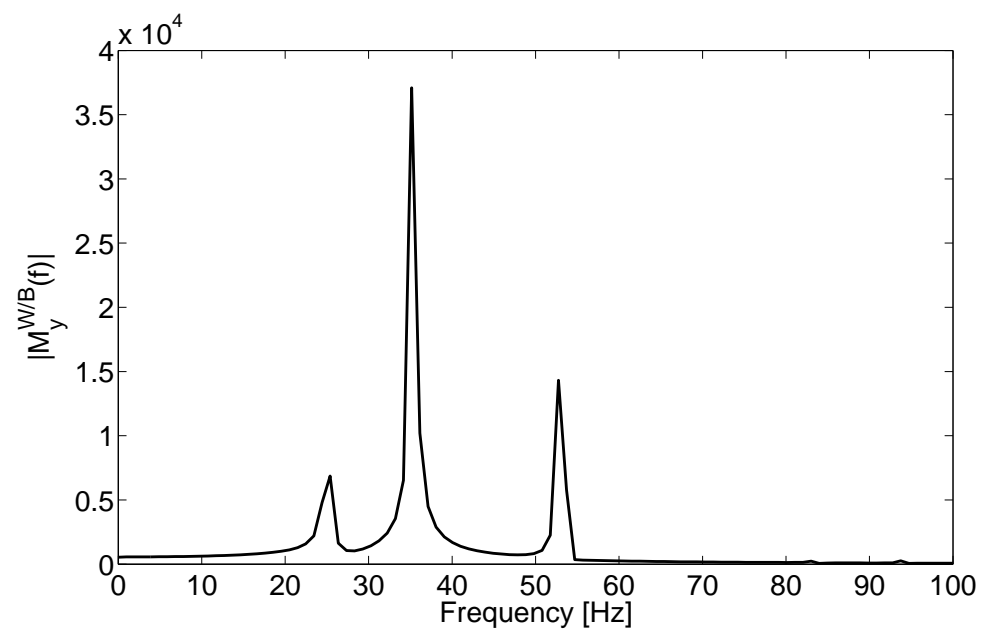

Figure 26. Frequency-domain response of $M_{y}^{W / B}$ for dynamic control surface case.

In addition to assessing overall vehicle response, another important consideration in the design and analysis of hypersonic vehicles is heating levels. While control surface inertial effects lead to oscillations in the forces and moments exerted by the control surface on the fuselage, such oscillations are not expected in the temperature-histories of the control surface due to the disparity between the aeroelastic and aerothermal time scales. Structural dynamic oscillations of the control surface lead to oscillations in the aeroheating boundary conditions at the outer surface. However, because the heat transfer process occurs on a slower time scale relative to the aeroelastic response, it is not expected that the time-histories of the nodal temperatures of the control surface will respond to such oscillations in the thermal boundary conditions. To verify these expectations, the minimum and maximum nodal temperatures over time are plotted in Fig. 28(a) and Fig. 28(b), respectively. Each figure gives the temperature time-history for both the dynamic and static control surface case in order to highlight the effect of control surface inertia on the temperature response. As expected, due to the disparate time scales of the aerothermal and aeroelastic processes, there exists no noticeable difference in the temperature response between the dynamic and static control surface cases for 


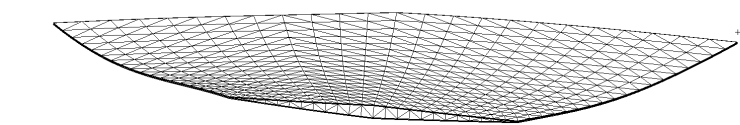

$z$

$X \int_{Y}$

(a) Mode 1: $25.1 \mathrm{~Hz}$.

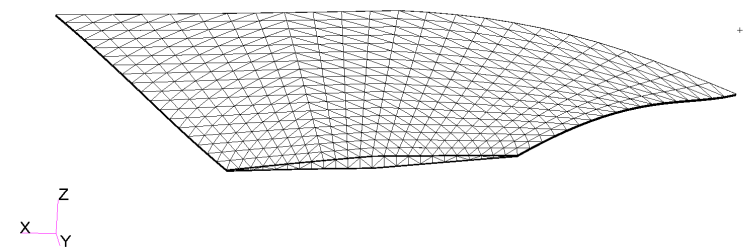

(c) Mode 3: $53.2 \mathrm{~Hz}$.

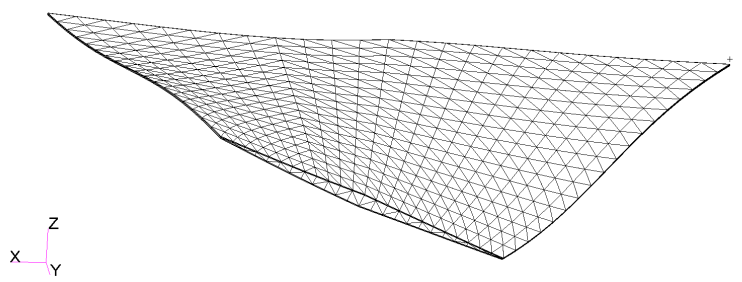

(b) Mode 2: $35.6 \mathrm{~Hz}$.

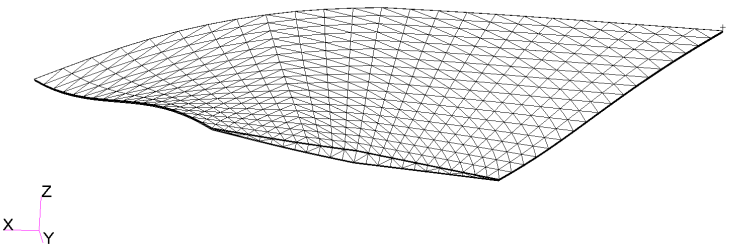

(d) Mode 4: $54.3 \mathrm{~Hz}$.

Figure 27. First four free vibration modes evaluated at thermal state obtained by heating structure for 400 s at $M_{\infty}=6.5, \alpha=0.1^{\circ}, \delta=0^{\circ}$, and $h=35 \mathrm{~km}$.

either the minimum and maximum temperatures. Thus, if one were solely interested in the evolution of temperature throughout the structure, these reults indicate that a quasi-static solution would be sufficient to capture the thermal response. Note that the initial slope of Fig. 28(a) is positive, while that of Fig. 28(b) is close to zero. This indicates that the maximum nodal temperature has approximately reached its steady state value at the end of the initial $400 \mathrm{~s}$ heating process, while the minimum temperatures are still increasing at this point. As expected, the increase in control surface deflection angle leads to an increase in dynamic pressure and therefore an increase in both the minimum and maximum nodal temperatures.

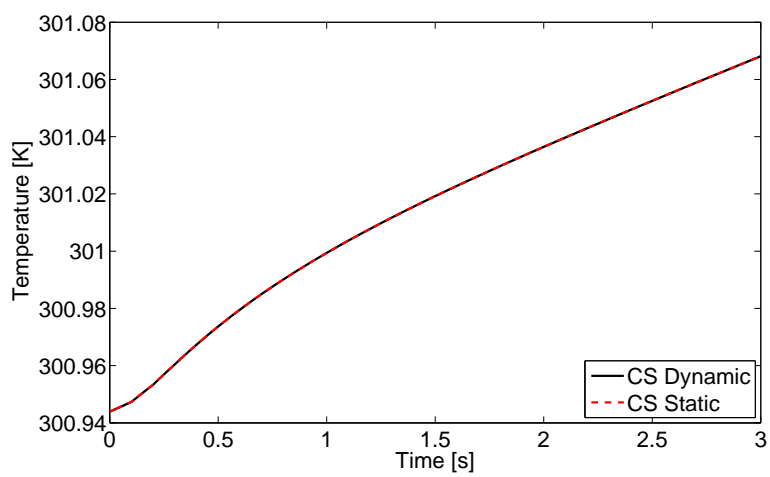

(a) Minimum nodal temperatures.

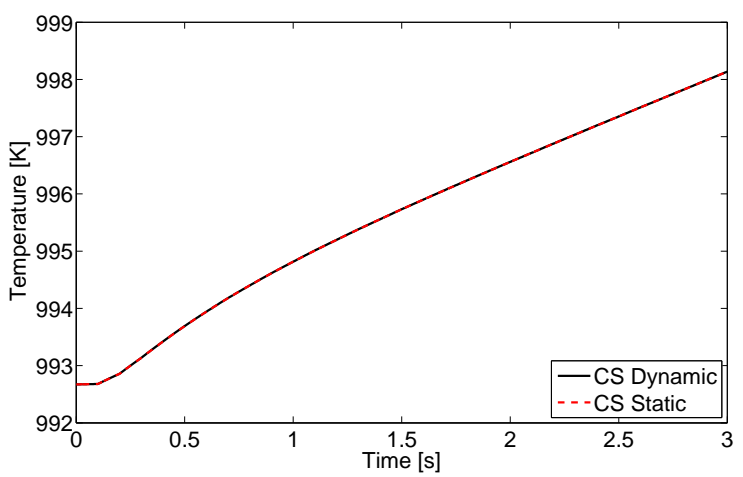

(b) Maximum nodal temperatures.

Figure 28. Minimum and maximum nodal temperatures over time for both static and dynamic control surface cases.

\section{VI.C.2. Commanded Step Increase and Decrease in Control Surface Deflection Angle}

The next case analyzed consists of a commanded step increase in $\delta$ of $3.9^{\circ}$ issued at $0.01 \mathrm{~s}$ into the transient and a subsequent command to return $\delta$ to $0^{\circ}$ issued at $0.4 \mathrm{~s}$ into the transient. The input command, $\delta_{\mathrm{cmd}}$, for this case is given by

$$
\delta_{\text {cmd }}(t)=20 H(t-0.01)\left[3.9^{\circ} \frac{\pi}{180^{\circ}}\right]+20 H(t-0.4)\left[-3.9^{\circ} \frac{\pi}{180^{\circ}}\right] .
$$

A plot showing $\delta(t)$ based on the input command of Eq. (39) is given in Fig. 29, where $\delta$ has been converted from radians to degrees for visualization purposes. As in the previous case, the flight conditions for this 


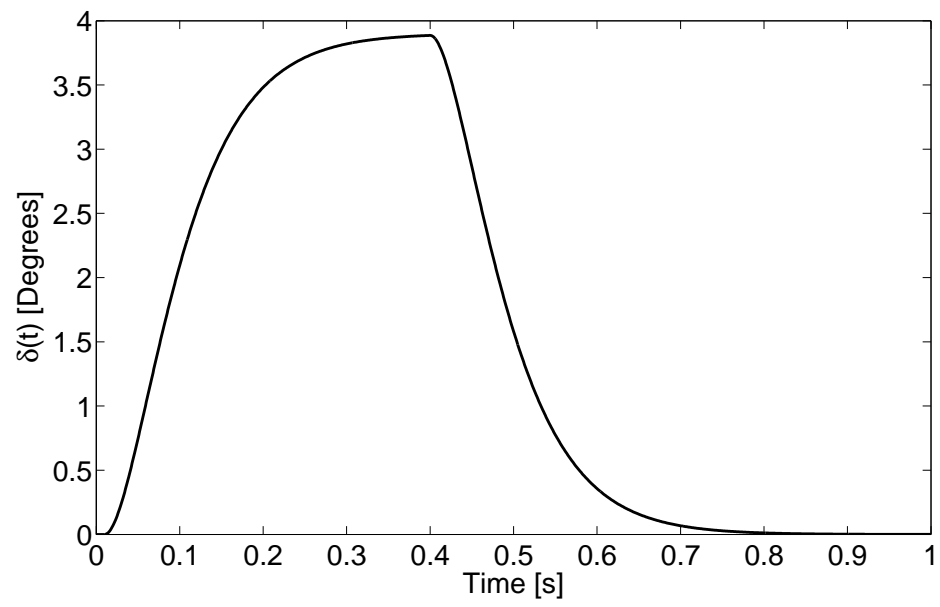

Figure 29. Time-history of applied control surface deflection angle, $\delta(t)$.

case are $M_{\infty}=6.5, h=35 \mathrm{~km}$, and $\alpha=0.1^{\circ}$. The time step sizes are: $\Delta t_{A E}=\Delta t_{H T}=0.001 \mathrm{~s}$ and $\Delta t_{A T}=0.1 \mathrm{~s}$. Again, the control surface is first heated at these flight conditions for $400 \mathrm{~s}$ at the initial control surface deflection angle, $\delta=0^{\circ}$. The control surface is then brought to aerothermoelastic equilibrium prior to beginning time-marching.

The entries of $x_{u}$ corresponding to the $z$ direction displacements of node 37 (located on the bottom surface, tip, leading edge) and node 475 (located on the bottom surface, tip, trailing edge) are given in Fig. 30(a) and Fig. 30(a), respectively, for both the dynamic and static control surface cases. Recall that $x_{u}$ contains both the constraint motion due to enforced rotation of the control surface about the hinge line and well as elastic deformation relative to the constraint motion. To remove the displacement components due to constraint motion, the elastic displacements, $x_{u}^{E}$, of nodes 37 and 475 are plotted in Fig. 31(a) and Fig. 31(b), respectively, for both the dynamic and static control surface cases. For the "CS Static" case in both figures, a slight increase in elastic displacement up to $0.4 \mathrm{~s}$ is observed as $\delta$ is increased and the aerodynamic pressure loads increase. As $\delta$ begins to return back to $0^{\circ}$ starting at 0.4 s, the aerodynamic pressure loads decrease and the elastic displacements therefore decrease slightly beginning at 0.4 s. The "CS Dynamic" results in both figures illustrate the effect of control surface structural dynamics which result in significant oscillations in the elastic displacements about the static values. For the time range considered, inclusion of control surface inertia results in elastic displacements of up to 1.9 times and 2.5 times the corresponding static elastic displacements for node 37 and 475 respectively.

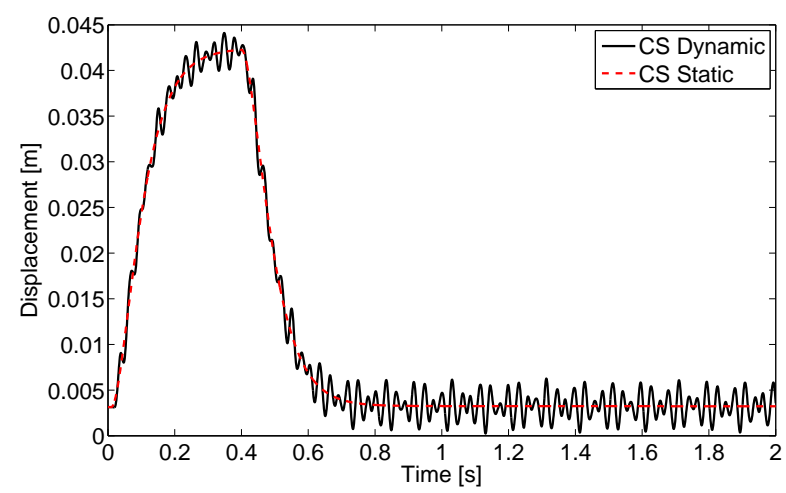

(a) Node 37 (bottom surface, tip, leading edge).

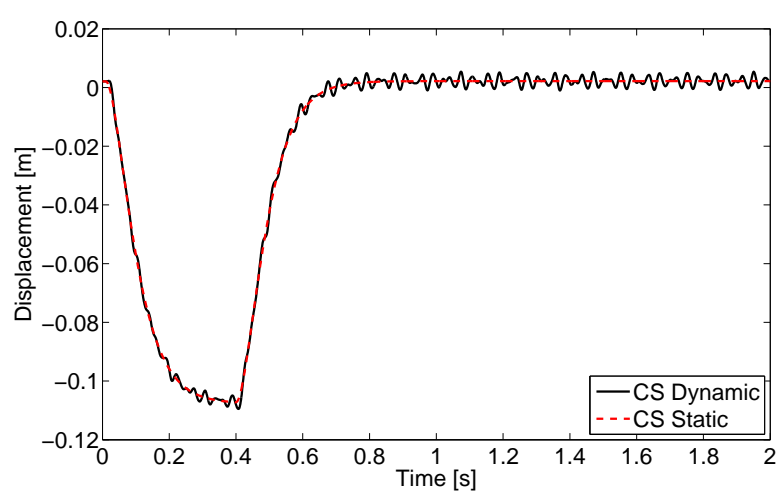

(b) Node 475 (bottom surface, tip, trailing edge).

Figure 30. Control surface total displacements, $x_{u}$, in $z$ direction under commanded change in deflection angle with and without control surface inertial effects for two selected nodes.

Plots of the pitching moment exerted by the control surface on the fuselage, $M_{y}^{W / B}$, are given in Fig. $32(\mathrm{a})$ 


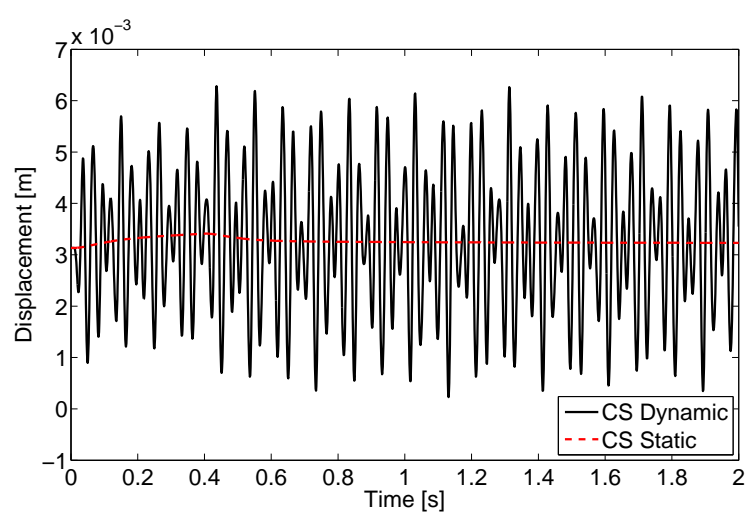

(a) Node 37 (bottom surface, tip, leading edge).

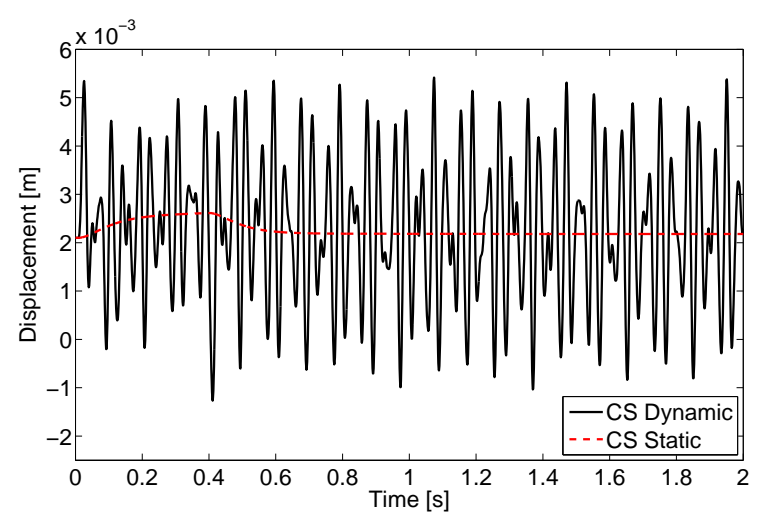

(b) Node 475 (bottom surface, tip, trailing edge).

Figure 31. Control surface elastic displacements, $x_{u}^{E}$, in $z$ direction under commanded change in deflection angle with and without control surface inertial effects.

and Fig. 32(b) for the static and dynamic control surface case, respectively. Examining Fig. 32(a), the static pitching moments results follow approximately the same trend as that of the time-history of $\delta$ given in Fig. 29. However, the static value of $M_{y}^{W / B}$ initially decreases when $\delta$ first begins to increase at initial time and initially increases when $\delta$ first begins to return back to $0^{\circ}$ at $0.4 \mathrm{~s}$. As discussed before, this effect is due to the unsteady aerodynamic loads induced by the instantaneous velocity of the control surface at these time instants. Additionally, slight discontinuities are observed in Fig. 32(a) in intervals of $0.1 \mathrm{~s}$ again due to the updating of the skin friction coefficients which is performed each time the thermal boundary conditions are updated. Examining Fig. 32(b), significant oscillations in $M_{y}^{W / B}$ are found to occur when control surface inertia is included. For the time range considered, the maximum absolute ratio of $M_{y}^{W / B}$ from the dynamic control surface case to that from the static control surface case is 528, again indicating the significant role of control surface inertia with regard to the pitching moment it generates on the fuselage.

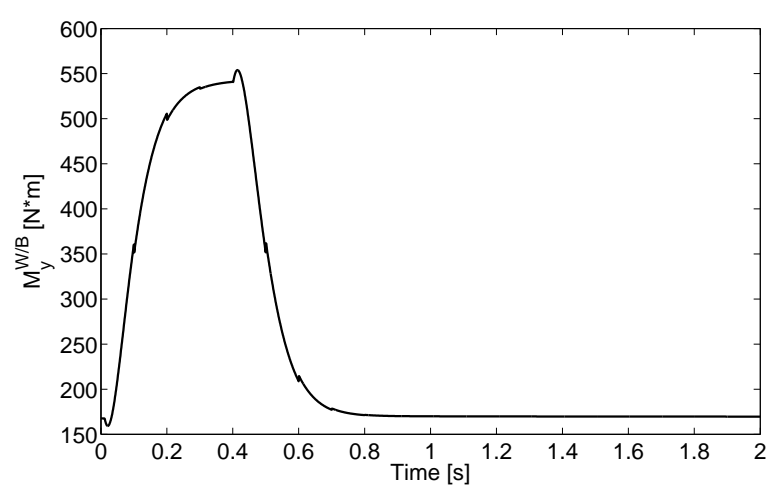

(a) Static control surface case.

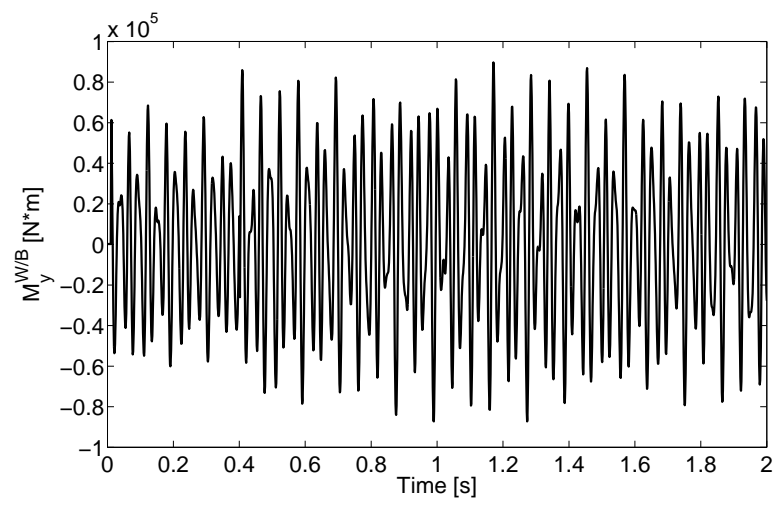

(b) Dynamic control surface case.

Figure 32. Time-domain response of $M_{y}^{W / B}$ for both static and dynamic control surface cases.

The minimum and maximum temperatures over time for this case are given in Fig. 33(a) and Fig. 33(b), respectively, for both the dynamic and static control surface cases. As in the previous section, there is not a noticeable difference in the temperature response when comparing the "CS Dynamic" case with the "CS Static" case, indicating that inertial effects do not impact the thermal response. The minimum temperature is monotonically increasing throughout the complete time-history shown in the plot. However, the rate of increase in the minimum temperature is not uniform throughout the transient due to the effect of the change in $\delta$. As $\delta$ begins to increase from $0^{\circ}$ to $3.9^{\circ}$, the minimum temperature begins to increase at a faster rate. Because the thermal boundary conditions are updated in intervals of $0.1 \mathrm{~s}$, though $\delta$ begins decreasing back to $0^{\circ}$ at $0.4 \mathrm{~s}$, the effect of the decreasing deflection angle on the minimum temperature is not observed until $0.5 \mathrm{~s}$. Thus, at approximately $0.5 \mathrm{~s}$, the minimum temperature begins increasing at a slower rate due to the 
decreased deflection angle. The maximum temperature remains nearly constant initially until the thermal boundary conditions are first updated at $0.1 \mathrm{~s}$, at which point it begins to increase due to the increase in $\delta$. At $0.5 \mathrm{~s}$, the rate of increase in the maximum temperature drops due to the fact that $\delta$ is decreasing at this point. At $0.6 \mathrm{~s}, \delta$ has nearly returned to $0^{\circ}$ and the maximum temperature begins decreasing slightly at this instant. Beyond approximately $1.2 \mathrm{~s}$, the maximum temperature begins increasing slightly likely due to the conduction of heat from lower temperature regions to higher temperature regions.

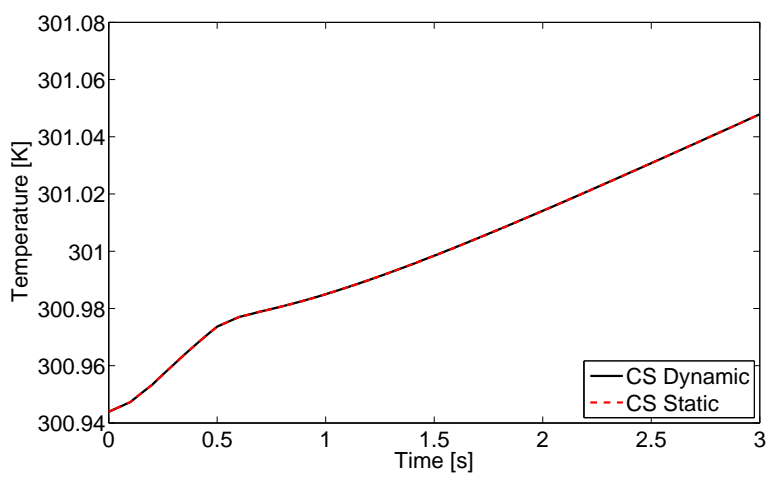

(a) Minimum nodal temperatures.

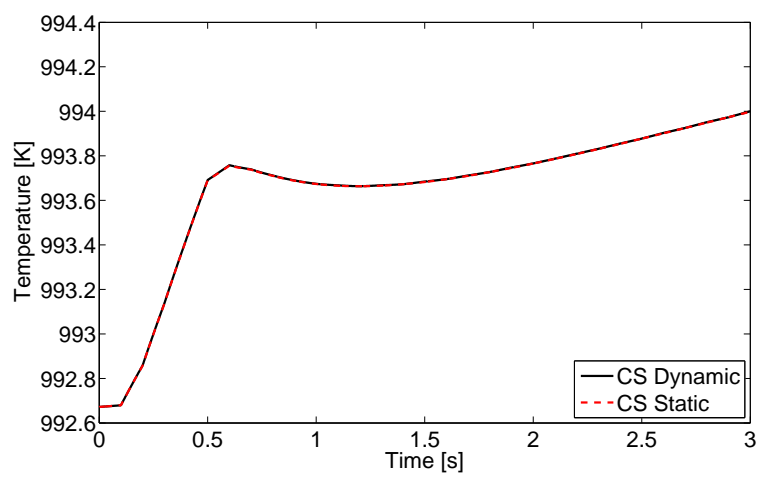

(b) Maximum nodal temperatures.

Figure 33. Minimum and maximum nodal temperatures over time for both static and dynamic control surface cases.

\section{Concluding Remarks}

This work investigates the impact of inertial loads due to control surface structural dynamics on overall vehicle response. Due to the dissimilar nature of the fuselage and control surface models used in the fullvehicle simulations, a direct monolithic coupling of these models is not easily facilitated. Thus, a partitioned solution methodology is presented in which the fuselage and control surface are each modeled independently and information is exchanged between the two within each aeroelastic time step to bring the systems into equilibrium. This approach is advantageous in that each component can be treated as a black box with respect to the other, and thus coupling of the models is reduced to input/output exchanges of information. As part of the partitioned solution approach described in this work, an iterative routine based on the secant method is outlined for efficiently bringing the two systems to equilibrium within each aeroelastic time step. Comparison of the partitioned approach with a monolithic approach demonstrates that the partitioned approach is mathematically equivalent to the monolithic system.

Using the partitioned solution scheme, the effect of control surface inertial loads on overall vehicle response was examined for a configuration in which the fuselage was represented as a single-DOF oscillator having a translational displacement degree of freedom in the $z$ direction. Comparison between a simulation which included control surface structural dynamics and a simulation which only used a static control surface solution showed that exclusion of control surface inertia results in an $11 \%$ overprediction in the frequency of the dominant fuselage oscillation component. It was also observed that the extent to which control surface inertia impacts the amplitude of fuselage oscillation is strongly dependent on the inertia of the fuselage itself. Examining the frequency content of the control surface response at one particular node, it was found that while control surface modes $1-5$ were all excited at least to a small extent, the most dominant modes in the control surface response were the fuselage mode and the first control surface mode. Additionally, the loads exerted by the control surface on the fuselage at the attachment point were investigated. Based on the results presented, exclusion of control surface inertia was found to result in an error in attachment point loads by up to a factor of 8 . However, the extent to which these attachment point loads impact fuselage response was found to be highly dependent on fuselage inertia. The frequency content of the attachment point loads showed that a $54.7 \mathrm{~Hz}$ mode had the greatest contribution to the loads. However, this mode did not significantly contribute to the fuselage response, indicating that the impact of attachment point loads on fuselage response is also depend on their frequency content.

In order to assess the impact of control surface inertial effects resulting from control surface rotation, a control input corresponding to control surface deflection angle was incorporated into the framework. An 
actuator model was used to convert the commanded change in deflection angle to the deflection angle applied by the actuator. Simulations were carried out for two different time-histories of the commanded control surface deflection angle. The first case consisted of a commanded step increase in control surface deflection angle. The second case consisted of a commanded step increase followed by a subsequent step decrease in control surface deflection angle. Both the total and elastic control surface displacements were examined for the cases of a static control surface solution and a dynamic one. Control surface inertia was found to result in an increase in the elastic displacements at one particular location by a factor of 1.9 for the first case and a factor of 2.5 for the second case with respect to the corresponding static solution. The resulting pitching moments exerted by the control surface on the fuselage through the attachment point were also examined. Results indicated that inclusion of control surface inertia results in departure of the instantaneous pitching moment from the control surface by up to a factor of 130 for the first case and 530 for the second case when compared against the static control surface solution. Analysis of the frequency content of the moment results indicated that the second control surface mode contributes most significantly to the oscillations of the moment about the static value. Thus, this mode is the most critical mode to control with respect to HSV pitching moment stabilization. Finally, assessment of the minimum and maximum temperatures over time for both cases demonstrated that the thermal response of the control surface is insensitive to structural dynamic oscillations due to the disparity between the aeroelastic and aerothermal time scales.

\section{Acknowledgment}

This work was supported by the Michigan-AFRL Collaborative Center in Control Science (MACCCS) under grant number FA 8650-07-2-3744 (Air Force Research Laboratory/Air Vehicles Directorate) with Michael Bolender as program manager.

\section{References}

${ }^{1}$ Anderson, Jr., J. D., Hypersonic and High-Temperature Gas Dynamics, McGraw-Hill, 1989.

${ }^{2}$ Bolender, M. A. and Doman, D. B., "Nonlinear Longitudinal Dynamical Model of an Air-Breathing Hypersonic Vehicle," Journal of Spacecraft and Rockets, Vol. 44, No. 2, March-April 2007, pp. 374-387.

${ }^{3}$ Chavez, F. R. and Schmidt, D. K., "Analytical Aeropropulsive/Aeroelastic Hypersonic-Vehicle Model with Dynamic Analysis," Journal of Guidance, Control, and Dynamics, Vol. 17, No. 6, March-April 2007, pp. 1308-1319. 1994.

${ }^{4}$ Bertin, J. J., Hypersonic Aerothermodynamics, American Institute of Aeronautics and Astronautics, Reston, Virgina,

${ }^{5}$ Schmidt, D. K., "Dynamics and Control of Hypersonic Aeropropulsive/Aeroelastic Vehicles," Proceedings of the 1992 AIAA Guidance, Navigation, and Control Conference, Hilton Head, South Carolina, AIAA 1992-4326.

${ }^{6}$ McRuer, D., "Design and Modeling Issues for Integrated Airframe/Propulsion Control of Hypersonic Flight Vehicles," Proceedings of the 1991 American Control Conference, Boston, MA, June 1991, pp. 729-734.

${ }^{7}$ Bilimoria, K. D. and Schmidt, D. K., "Integrated Development of the Equations of Motion for Elastic Hypersonic Vehicles," Journal of Guidance, Control, and Dynamics, Vol. 18, No. 1, January-February 1995, pp. 73-81.

${ }^{8}$ Parker, J. T., Serrani, A., Yurkovich, S., Bolender, M. A., and Doman, D. B., "Control-Oriented Modeling of an AirBreathing Hypersonic Vehicle," Journal of Guidance, Control, and Dynamics, Vol. 30, No. 3, May-June 2007, pp. 856-869.

${ }^{9}$ Bolender, M. A., "An Overview on Dynamics and Controls Modelling of Hypersonic Vehicles," Proceedings of the 2009 American Control Conference, St. Louis, MO, June 2009, pp. 2507-2512.

${ }^{10}$ Frendreis, S. G. V. and Cesnik, C. E. S., "3D Simulation of a Flexible Hypersonic Vehicle," Proceedings of the 2010 AIAA Atmospheric Flight Mechanics Conference, Toronto, Ontario, Canada, AIAA 2010-8229.

${ }^{11}$ Falkiewicz, N. J. and Cesnik, C. E. S., "A Reduced-Order Modeling Framework for Integrated Thermo-Elastic Analysis of Hypersonic Vehicles," Proceedings of the 50th AIAA/ASME/ASCE/AHS/ASC Structures, Structural Dynamics, and Materials Conference, Palm Springs, California, AIAA 2009-2308.

${ }^{12}$ Falkiewicz, N. J. and Cesnik, C. E. S., "Proper Orthogonal Decomposition for Reduced-Order Thermal Solution in Hypersonic Aerothermoelastic Simulations," AIAA Journal, Vol. 49, No. 5, May 2011.

${ }^{13}$ Falkiewicz, N. J., Cesnik, C. E. S., Bolender, M. A., and Doman, D. B., "Thermoelastic Formulation of a Hypersonic Vehicle Control Surface for Control-Oriented Simulation," Proceedings of the 2009 AIAA Guidance, Navigation, and Control Conference, Chicago, Illinois, AIAA 2009-6284.

${ }^{14}$ Falkiewicz, N. J., Cesnik, C. E. S., Crowell, A. R., and McNamara, J. J., "Reduced-Order Aerothermoelastic Framework for Hypersonic Vehicle Control Simulation," AIAA Journal, Vol. 49, No. 8, August 2011.

${ }^{15}$ Falkiewicz, N. J. and Cesnik, C. E. S., "Enhanced Modal Solutions for Structural Dynamics in Aerothermoelastic Analysis," Proceedings of the 52nd AIAA/ASME/ASCE/AHS/ASC Structures, Structural Dynamics, and Materials Conference, Denver, Colorado, AIAA 2011-1963.

${ }^{16}$ Eckert, E. R. G., "Engineering Relations for Friction and Heat Transfer to Surfaces in High Velocity Flow," Journal of the Aeronautical Sciences, Vol. 22, No. 8, March 23, 1955, pp. 585-587. 
${ }^{17}$ Vogel, J. M., Kelkar, A. G., Inger, G., Whitmer, C., Sidlinger, A., and Rodriguez, A., "Control-Relevant Modeling of Hypersonic Vehicles," Proceedings of the 2009 American Control Conference, St. Louis, MO, June 2009, pp. $2519-2524$.

${ }^{18}$ Pendleton, E., Moster, G., and Keller, D., "Transonic Aeroelastic Models of Highly Swept Hypersonic Lifting Surfaces," Journal of Aircraft, Vol. 32, No. 6, Nov. - Dec. 1995, pp. 1169-1176.

${ }^{19}$ Oppenheimer, M. W. and Doman, D. B., "A Hypersonic Vehicle Model Developed with Piston Theory," Proceedings of the 2006 AIAA Atmospheric Flight Mechanics Conference, Keystone, Colorado, AIAA 2006-6637.

${ }^{20} \mathrm{McNamara}$, J. J., Aeroelastic and Aerothermoelastic Behavior of Two and Three Dimensional Lifting Surfaces in Hypersonic Flow, Ph.D. thesis, University of Michigan, Ann Arbor, Michigan, 2005.

${ }^{21}$ Shih, P., Prunty, J., and Mueller, R., "Thermostructural Concepts for Hypervelocity Vehicles," Journal of Aircraft, Vol. 28, No. 5, May 1991, pp. 337-345.

${ }^{22}$ Myers, D., Martin, C., and Blosser, M., "Parametric Weight Comparison of Advanced Metallic, Ceramic Tile, and Ceramic Blanket Thermal Protection Systems," Tech. Rep. NASA TM-210289, Langley Research Center, June 2000.

${ }^{23}$ Wieting, A., Dechaumphai, P., Bey, K., Thornton, E., and Morgan, K., "Application of Integrated Fluid-ThermalStructural Analysis Methods," Thin-Walled Structures, Vol. 11, No. 1-2, 1991, pp. 1-23.

${ }^{24}$ Blevins, R., Bofilios, D., Holehouse, I., Hwa, V., Tratt, M., Laganelli, A., Pozefsky, P., and Pierucii, M., "Thermo-VibroAcoustic Loads and Fatigue of Hypersonic Flight Vehicle Structure," Tech. Rep. AFRL-RB-WP-TR-2009-3139, Air Force Research Laboratory, June 2009.

${ }^{25}$ Ellis, D., Pagel, L., and Schaeffer, D., "Design and Fabrication of a Radiative Actively Cooled Honeycomb Sandwich Structural Panel for a Hypersonic Aircraft," Tech. Rep. NASA-CR-2957, NASA, March 1978.

${ }^{26}$ Leyens, C. and Peters, M., Titanium and Titanium Alloys, Wiley-VHC, 2003.

${ }^{27}$ Department of Defense, Military Handbook - MIL-HDBK-5H: Metallic Materials and Elements for Aerospace Vehicle Structures, December 1998.

${ }^{28}$ Welsch, G., Boyer, R., and Collings, E. W., Materials Properties Handbook: Titanium Alloys, ASM International, 1994.

${ }^{29}$ Bolender, M. A. and Doman, D. B., "Nonlinear Longitudinal Dynamical Model of an Air-Breathing Hypersonic Vehicle," Journal of Spacecraft and Rockets, Vol. 44, No. 2, 2007, pp. 374-387.

${ }^{30}$ Dalle, D. J., Frendreis, S. G. V., Driscoll, J. F., and Cesnik, C. E. S., "Hypersonic Vehicle Flight Dynamics with Coupled Aerodynamics and Reduced-order Propulsive Models," Proceedings of the 2010 AIAA Atmospheric Flight Mechanics Conference, Toronto, Ontario, Canada, AIAA 2010-7930.

${ }^{31}$ Falkiewicz, N. J. and Cesnik, C. E. S., "Proper Orthogonal Decomposition for Reduced-Order Thermal Solution in Hypersonic Aerothermoelastic Simulations," Proceedings of the 51st AIAA/ASME/ASCE/AHS/ASC Structures, Structural Dynamics, and Materials Conference, Orlando, Florida, AIAA 2010-2798.

${ }^{32}$ Wilson, E. L., Yuan, M.-W., and Dickens, J. M., "Dynamic Analysis by Direct Superposition of Ritz Vectors," Earthquake Engineering \&5 Structural Dynamics, Vol. 10, No. 6, Nov. - Dec. 1982, pp. 813-821.

${ }^{33}$ Craig, R. R. and Kurdila, A. J., Fundamentals of Structural Dynamics, John Wiley \& Sons, Inc., 2nd ed., 2006.

${ }^{34}$ MSC.Software Corporation, Santa Ana, CA, MSC.Nastran Basic Dynamic Analysis User's Guide: Version 68, 2004.

${ }^{35}$ Heath, M. T., Scientific Computing : An Introductory Survey, McGraw-Hill, 2nd ed., 2002.

${ }^{36}$ Crowell, A. R., McNamara, J. J., Kecskemety, K. M., and Goerig, T. W., "A Reduced Order Aerothermodynamic Modeling Framework for Hypersonic Aerothermoelasticity," Proceedings of the 51st AIAA/ASME/ASCE/AHS/ASC Structures, Structural Dynamics, and Materials Conference, Orlando, Florida, AIAA 2010-2969.

${ }^{37}$ Gibson, T. E., Crespo, L. G., and Annaswamy, A. M., "Adaptive Control of Hypersonic Vehicles in the Presence of Modeling Uncertainties," Proceedings of the 2009 American Control Conference, St. Louis, MO, June 2009, pp. 3178-3183. 